\title{
Splitting of a Set of Equivalent Sites in Centrosymmetric Space Groups Into Subsets Under Homogeneous Stress
}

\author{
J. B. Wachtman, Jr., and H. S. Peiser
}

(November 18, 1964)

\begin{abstract}
It is assumed that the symmetry operations possessed by a homogeneously stressed crystal will be those common to the crystal and to the macroscopic state of stress. Application of stress either leaves a space group unaltered or lowers it to a subgroup. Such lowering can always be considered to take place in successive steps each of which leaves no group intermediate between the starting group and the subgroup. Each such step can be accomplished by a uniaxial stress; for the centrosymmetric space groups, all but one of the symmetry reductions consisting of two or more successive steps can also be accomplished by a uniaxial stress. A set of sites all of which are equivalent in the unstrained crystal may split into two or more subsets under stress. For each space group all possibilities are taken into account by considering the behavior of the general position (most general set of symmetry related sites) because the behavior of each special position can easily be derived by specializing the description of the general position. The splitting of the general position under homogeneous stress is tabulated for all possibilities for each centrosymmetric space group.
\end{abstract}

\section{Introduction}

The reduction of the symmetry of crystals and the accompanying introduction of inequivalence of sites within crystals is not only a subject of interest for its own sake, but is also important in several areas of solid state physics. The present writers became interested from the standpoint of internal friction studies. In such studies, stress is applied to a crystal to lower its symmetry and cause a partial ordering of defects. This subject has been developed extensively $[1-5]^{1}$ and other possible applications of stress-induced symmetry lowering have also been discussed [6]. The present paper is limited to a tabulation of the splitting of sets of initially equivalent sites into subsets under homogeneous stress.

\section{Specification of Stress or Strain}

It is possible to formulate the present paper in terms of either stress or strain, but stress appears to be somewhat more appropriate as we shall subsequently point out in connection with Curie's principle. We restrict consideration to homogeneous stress; i.e., to stress which varies with location slowly enough that it may be assumed constant over a number of unit cells.

Stress is a second-rank, symmetric tensor [7] and therefore has six independent components in an arbitrary rectangular Cartesian coordinate system. For any given state of stress, a rotated coordinate system (called the principal axis system and designated $X, Y, Z)$ can be found in which the stress can be described by at most three nonzero components; i.e., the stress tensor is diagonalized. The six independent components are thus specified by three angles giving the orientation of the principal axis

1 Figures in brackets indicate the literature references at the end of this paper. system and three diagonal components in this system. Any state of homogeneous stress can then be characterized as being uniaxial, biaxial, or triaxial according to the number of nonzero components in the principal axis system. This specification of the six quantities describing stress (or any other symmetric second-rank tensor) can be visualized in terms of the orientation, shape, and size of the representation quartic. If $\sigma_{i j}$ denotes the components of the stress in a set of rectangular Cartesian coordinates $x_{i}$ and if we define a surface made up of points $\left(x_{1}\right.$, $x_{2}, x_{3}$ ) satisfying

$$
\sum_{i, j=1}^{3} \sigma_{i j} x_{i} x_{j}=1
$$

we obtain a surface called the representation quartic. If we transform the stress to the principal axis system, all cross terms vanish. It is then easy to see that, if the three components of stress in the principal axis system are all larger than zero, the surface is an ellipsoid. Cases where one, two, or all three of the principal stresses are negative correspond respectively to a hyperboloid of one sheet, a hyperboloid of two sheets, and to an imaginary ellipsoid [7]. It is, however, not necessary to consider negative stress components in this paper because we are concerned only with the symmetry of the stress and this symmetry is unchanged if one or more of the principal stresses change sign.

We can thus describe any triaxial stress by giving the shape, size, and orientation of an ellipsoid. This description has the advantage of ease of visualization, but requires us to specialize to fewer dimensions for biaxial or uniaxial stress; the appropriate figures are an ellipse and the two ends of a straight line segment respectively. We can retain the advantage of easy visualization and deal only with three-dimensional figures by introducing the modified stress ellipsoid 
which is the figure obtained by adding an arbitrary value of isostatic stress to the state of stress being considered. This addition is permissible in dealing with symmetry changes caused by stress because an isostatic stress should cause no change of crystal symmetry. The modified stress ellipsoid for uniaxial stress is thus an ellipsoid of revolution with its unique axis pointing in the direction of the uniaxial stress. The modified stress ellipsoid for biaxial stress is one with three unequal axes as is the modified stress ellipsoid for triaxial stress; these two ellipsoids can be distinguished only by comparing the size of the principal axes with the value of the assumed isostatic stress. The fact that one cannot distinguish between biaxial and triaxial stress from the shape of the modified stress ellipsoid alone causes no difficulty in the present application because any reduction of crystal symmetry that can be caused by stress can be accomplished by biaxial stress. Isostatic stress can lead to mechanical relaxation by changing the degree of inequivalence of sites that are already inequivalent. This is illustrated, for example, by the isostatic relaxation modes discussed by Franklin [8]. Such cases do not concern us here because we are restricting consideration to inequivalence of sites that are equivalent in the absence of stress.

Stresses applied to a given crystal can be classified into three types depending on the effect on the symmetry of the crystal. First, and most highly specialized, is stress that causes no reduction of symmetry. This is the type in which the modified stress ellipsoid has all of the symmetry elements of the crystal and has them in the same orientation as the crystal. Examples are isostatic stress applied to any crystal or uniaxial stress applied parallel to the unique axis of a tetragonal, hexagonal, or trigonal crystal. Second, and less specialized, is stress that causes a minimum step of symmetry lowering; that is, symmetry lowering such that there is no space group which is a stressinduced subgroup of the original group, which is also a supergroup of the final group, and which is distinct from both initial and final groups. Third, and least specialized, is stress that causes a compound step of symmetry lowering, i.e., which does leave one or more distinct space groups intermediate between the initial and final space groups. In this paper, we specify the stress for all the possible minimum steps and compound steps that can be caused by homogeneous stress.

In specifying stress, we need only specify (1) whether two of the axes $X, Y, Z$ of the modified stress ellipsoid are equal (if so, $Z$ is taken as the unique axis) and (2) the orientation of these axes. In giving these specifications, we adopt the convention that there is no equality of axes and no specialization of orientation unless explicitly stated. Our intention in this paper is to give the most general (i.e., least specialized) stress that will accomplish each symmetry reduction. From an experimental point of view, one might wish to know what is the most highly specialized stress that can be used, because it is easier to apply uniaxial stress than biaxial stress. We shall leave detailed treatment of this subject to a subsequent paper and simply state the result that any minimum step can be done by uniaxial stress. For the centrosymmetric space groups, any compound step can also be done by uniaxial stress, with the exception that biaxial stress is required to reach space groups associated with one of the two nonequivalent orientations in which mmm occurs as a subgroup of $\mathrm{m} 3 \mathrm{~m}$.

The strain can be specified by an ellipsoid in exactly the same way as the stress and a modified strain ellipsoid can be introduced to correspond to the modified stress ellipsoid. For an elastically isotropic body, the principal axes of the modified strain ellipsoid have the same orientation as the principal axes of the modified stress ellipsoid. Crystals are usually elastically anisotropic, however, and the principal axes of the two modified ellipsoids need not be parallel. The lowering of crystal symmetry can, if desired, be discussed in terms of the modified stress ellipsoid without error because we are concerned only with the retention of symmetry by the stressed crystal. To be retained a symmetry element must belong to the unstressed crystal and the modified stress ellipsoid (Curie's principle [6]); it must therefore also be possessed by the strain ellipsoid. We could thus use the modified strain ellipsoid in our discussion, but it is conceptually simpler to use the modified stress ellipsoid.

\section{Effect of Symmetry Lowering on Space Groups}

\subsection{Reduction of Point-Group Symmetry}

The point groups are discussed in this paper primarily because the stress required to lower a given space group to a subgroup can be specified completely in terms of the corresponding point group, i.e., the specification is the same for any space-group reduction corresponding to the reduction from one point group to another. This permits the spacegroup results to be collected into tables, each of which consists of all transitions from one space group to another associated with reduction from a given point group to another. The stress can thus be described once for each table provided the specification is given in terms of axes that are fixed with respect to the point group. For two space groups associated with the same point group the axes used in the International Tables [9] are not always taken in the same orientation relative to the point groups. In the centrosymmetric space goups this complication arises only for $\overline{3} \mathrm{~m}$. For all tables other than table 8 each specification of stress is the same for all of the space groups in that table.

As noted in the preceding section, we are giving the most general specification of stress, despite the fact that more specialized stresses may be of interest in some applications. Such specializations can be discussed in terms of the point groups alone and so are being left for treatment in a paper devoted to point groups. 


\subsection{Reduction of Space-Group Symmetry}

In considering the subgroup reached from a given space group, we again assume that the subgroup has the symmetry common to the starting group and the stress. For the symmorphic space groups (those which contain their point groups), we can again take the symmetry of the stress to be that of the modified stress ellipsoid, but for the nonsymmorphic space groups, we must extend our understanding of "symmetry of the stress" to include all glide planes parallel to mirror planes of the modified stress ellipsoid and all screw axes parallel to rotation axes of the same order possessed by the modified stress ellipsoid. The possible stress-induced reductions of space groups have been tabulated [6].

\subsection{Mapping of General Positions}

A general position (set of symmetry-related but unspecialized sites) in a starting space group must remain a general position in a subgroup because no symmetry can be gained by application of stress. The number of sites in the general position per lattice point in the starting space group is equal to the order, $N$, of the point group associated with the starting space group. In going to a subgroup with associated point group of order $n$, this set of $N$ sites must split into $N / n$ subsets, each of which contains $n$ sites and is a general position of the final space group. Table 1 through 12 give the coordinates (referred to the axes of the starting space group) of these subsets. In these tables, we have used the coordinates of the International Tables [9]; where a choice of origin is given, we have taken the origin at a center of symmetry in order to permit coordinates with \pm prefix to indicate a pair of sites. For the rhombohedral space groups, we have used hexagonal axes despite the resulting triple cell, because hexagonal axes seem to be preferred by most workers.

To illustrate the use of tables 1 through 12 consider, for example, space group $\mathrm{P} 2 / \mathrm{m}$ associated with point group $2 / \mathrm{m}$, which goes to space group P $\overline{1}$ associated with point group $\overline{1}$ under the action of a stress that is not specially oriented to preserve the $2 / \mathrm{m}$ symmetry. In $\mathrm{P} 2 / \mathrm{m}$, the general site $(x, y, z)$ is carried by the symmetry operations into the equivalent sites $(-x,-y,-z),(+x,-y,+z)$, and $(-x,+y,-z)$. Under the action of the symmetry operations remaining in $\mathrm{P} \overline{1}$, the site $(x, y, z)$ is carried only into $(-x,-y,-z)$ and these two sites form a general position in $\mathrm{P} \overline{1}$. The site $(+x,-y$, $+z)$ is equivalent to $(-x,+y,-z)$ and these two together also form a general position in $\mathrm{P} \overline{1}$. This mapping of the complete set of four equivalent sites making up a general position in $\mathrm{P} 2 / \mathrm{m}$ onto two distinct subsets each of which composes a general position in $\mathrm{P}_{1}$ is indicated in the tables by listing the sites making up a general position for $\mathrm{P} 2 / \mathrm{m}$ in the two subsets $\{ \pm(x, y, z)\}$ and $\{ \pm(x, \bar{y}, z)\}$.

Note that the coordinates $(x, y, z)$ of a site have one set of values $\left(x_{1}, y_{1}, z_{1}\right)$ in the first subset and another set of values $\left(x_{2}, y_{2}, z_{2}\right)$ in the second subset. This is true throughout the tables; whenever a set of equivalent sites splits into subsets, the coordinates of any site in the stressed crystal are related by symmetry to those of the other sites in the same subset, but are not so related to the coordinates of sites in other subsets, and are distinct from the original coordinates in the unstressed crystal. This distinction is brought out in the tables by keeping the coordinates of sites in different subsets in different columns rather than by using subscripts.

A given point group may occur in more than one orientation as a subgroup of an initial point group. These two settings may be equivalent (i.e., one is generated from the other by a symmetry operation of the initial point group) or inequivalent. Equivalent settings of subgroups of point groups correspond to equivalent settings of subgroups of space groups and need not be considered separately. Inequivalent settings correspond to physically distinct symmetry lowerings and are listed separately in the tables. See, for example, the two inequivalent ways in which mmm can occur as a subgroup of $4 / \mathrm{mmm}$ (both given in one table, 6 , by use of top and bottom captions) and the three ways $2 / \mathrm{m}$ can result from $\mathrm{mmm}$. The occurrence of two or more inequivalent settings of a subgroup can be shown by two or more parallel tie lines on a decomposition chart, as was done for the point groups by the Internationale Tabellen [10].

A second example will illustrate both the possibility of alternative space groups associated with the same point group and the situation which arises when the number of lattice points per cell changes: consider the behavior of $\mathrm{Cmmm}$ under a stress oriented to lower $\mathrm{mmm}$ to $2 / \mathrm{m}$. This reduction can be done by letting one axis of the modified stress ellipsoid coincide with any one of the three diad axes in mmm. We use the convention of the International Tables [9] and take our $x, y, z$ axes in the same orientation relative to the space group operations, i.e., $x$ and $y$ axes in the plane of the diagram as shown in the International Tables with origin at upper left-hand corner. The positive direction of the $x$ axis is downward in the page and that of the $y$ axis is to the right in the page, the interaxial angle is determined by the symmetry. The positive $z$ axis points upward from the page; its angular relations to the other two axes are determined by symmetry. If one axis of the modified stress ellipsoid is taken parallel to either the $x$ or $y$ axis of $\mathrm{Cmmm}$ and otherwise unspecialized the space group $\mathrm{C} 2 / \mathrm{m}$ is obtained. If, however, an axis of the modified stress ellipsoid is taken parallel to $z$, the space group $\mathrm{P} 2 / \mathrm{m}$ is obtained. We have gone from a face-centered cell with two lattice points per cell to a cell with one lattice point. This causes no difficulty in the tables, however, because we list the sites associated with a lattice point rather than a cell. Thus, the set of general sites in Cmmm splits into the subsets $\{ \pm(x, y, z), \pm(x, y, \bar{z})\}$ and $\{ \pm(x, \bar{y}, \bar{z})$, $\pm(x, \bar{y}, z)\}$. Each of these subsets must map onto the set of sites making up the general position of 
TABLE 1. Reduction from $m 3 m$ to $4 / \mathrm{mmm}$

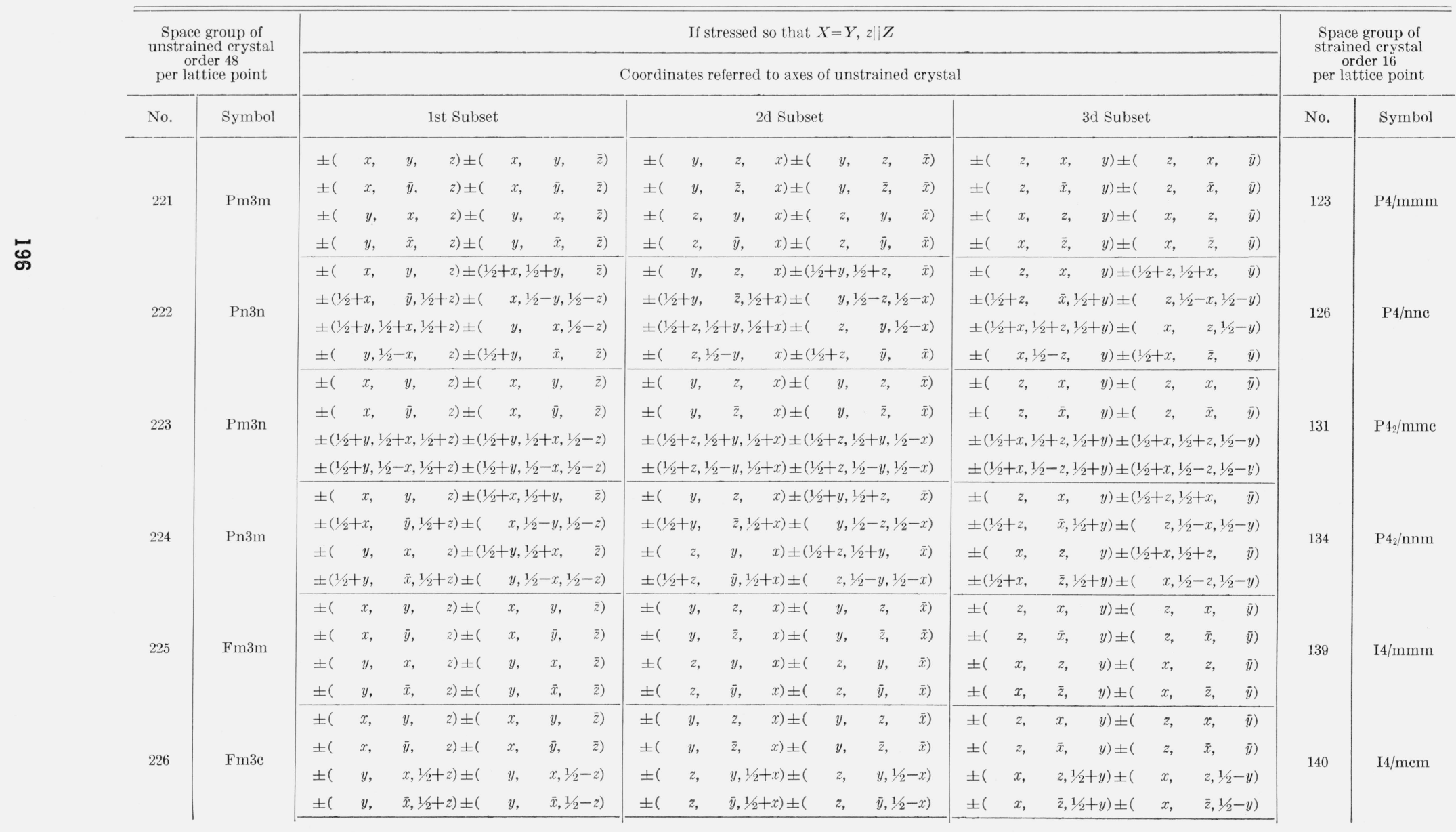




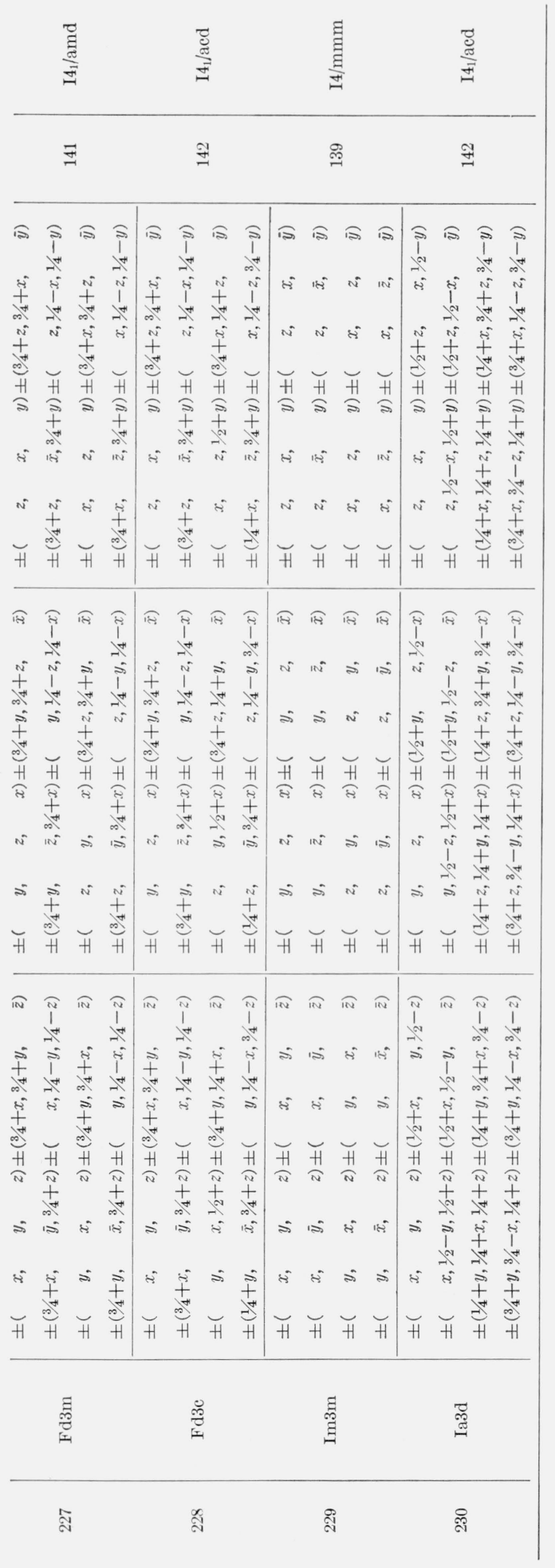

$\mathrm{P} 2 / \mathrm{m}$ which we have previously written as $\{ \pm(x, y, z), \pm(x, \bar{y}, z)\}$. The apparent discrepancy between $\{ \pm(x, y, z), \pm(x, y, \bar{z})\}$ and $\{ \pm(x, y, z)$, $\pm(x, \bar{y}, z)\}$ results from having the former expressed in the axes of $\mathrm{Cmmm}$ and the latter in the axes of $\mathrm{P} 2 / \mathrm{m}$; in going from one to the other, the roles of the $y$ and $z$ axes have been interchanged. This example emphasizes the fact that the present tables give the subsets in terms of the axes of the starting space group and that the relation of these to the axes of the final space group must be considered if one wishes to express the results in terms of the latter axes. The latter may differ from the former not only in orientation, but also in a shift of the origin.

The above two examples taken together illustrate the result of going directly from $\mathrm{Cmmm}$ to $\mathrm{P} \overline{1}$. Combining the steps from Cmmm to $\mathrm{P} 2 / \mathrm{m}$ and from $\mathrm{P} 2 / \mathrm{m}$ to $\mathrm{P} \overline{1}$ and allowing for the change of axes, we see that the set of sites per lattice point making up the general position in Cmmm splits into four subsets $\{ \pm(x, y, z)\},\{ \pm(x, y, \bar{z})\},\{ \pm(x, \bar{y}, z)\}$, and $\{ \pm(x, \bar{y}, \bar{z})\}$.

The most general stress that will cause a compound step of symmetry reduction is simply the most general stress required for the last minimum step. Specifying the conditions on the axes $X, Y, Z$ of the modified stress ellipsoid in terms of the axes of a super group more than one minimum step above the final space group requires consideration of the relation of the axes of the minimum-step supergroup to those of the starting supergroup. This could be done in a formal manner by considering the axis transformations, but it is easier to omit consideration of the intermediate steps and write down the stress specification in terms of the axes of the starting point group by direct inspection of the remaining symmetry. The results are summarized in table 13 .

\subsection{Mapping of Special Positions}

The coordinates given in tables 1 through 12 are those of the sites in the general positions, i.e, the sites that are not left invariant by any symmetry operation other than the identity. The special positions, listed in the International Tables for each space group, are those which possess (are left invariant by) a group of symmetry operations. The behavior of each of the special positions is easily derived from that of the general position. For example, in the transition of $\mathrm{Cmmm}$ to $\mathrm{P} 2 / \mathrm{m}$ which we previously discussed, we may specialize the general site $(x, y, z)$ to $(x, y, 0)$ so that the site lies on the horizontal mirror plane, $z=0$. Action of the symmetry operations on this site produces a complete set of four equivalent special sites (of this type) per lattice point so that there is a two-to-one mapping of the eight sites in the general position onto the four sites of the special position in the unstrained crystal. By performing this mapping (in this case, setting $z=0$ ) in the stress-induced subsets, we can arrive at the stress-induced splittting for the special position. Thus, we find that the one four-fold stress-induced subset of a general position $\{ \pm(x, y$, 
TABLE 2. Reduction from $m 3 m$ to $\overline{3} m$

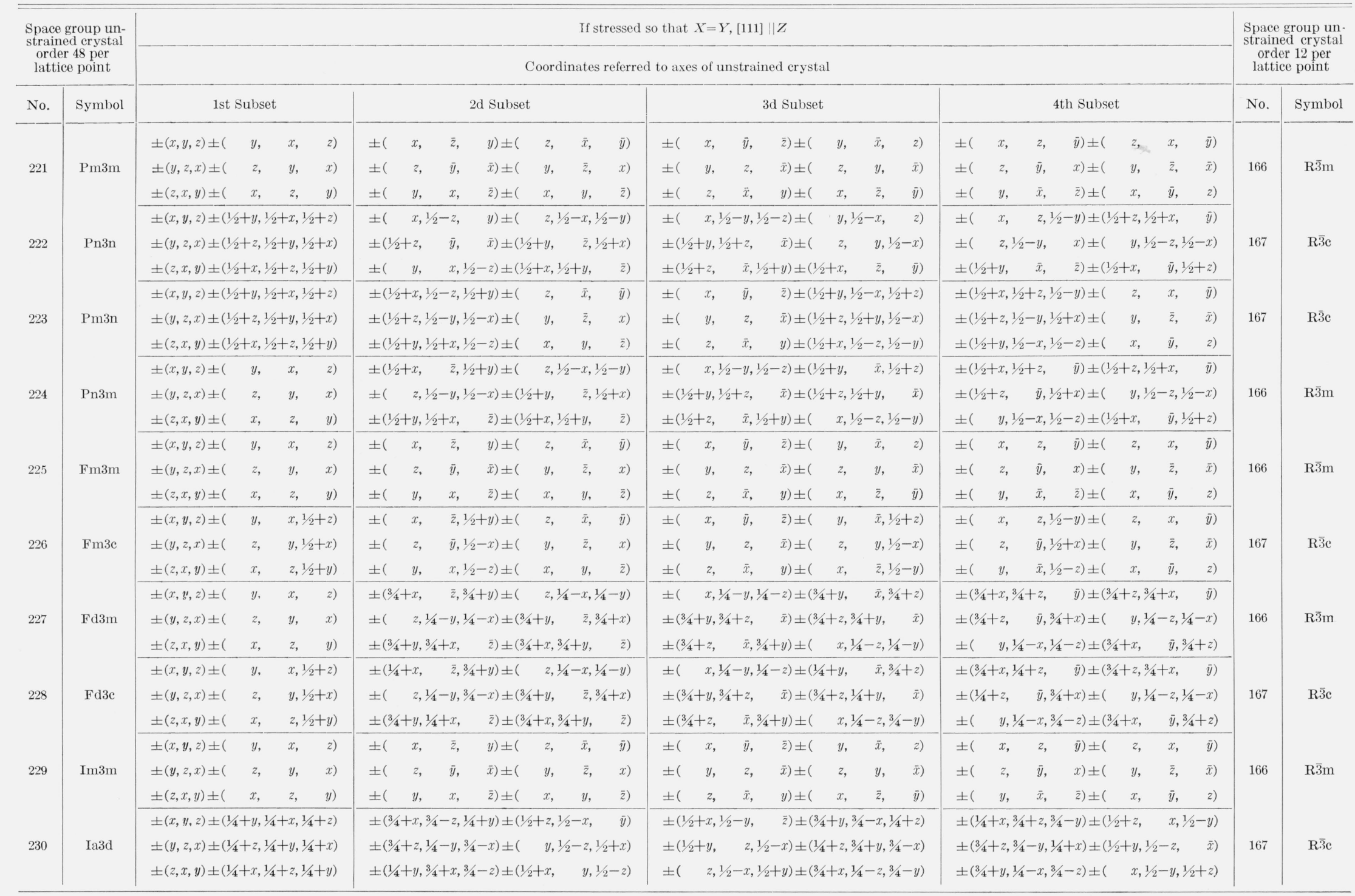


TABLE 3. Reduction from $\mathrm{m} 3$ to $\mathrm{mmm}$

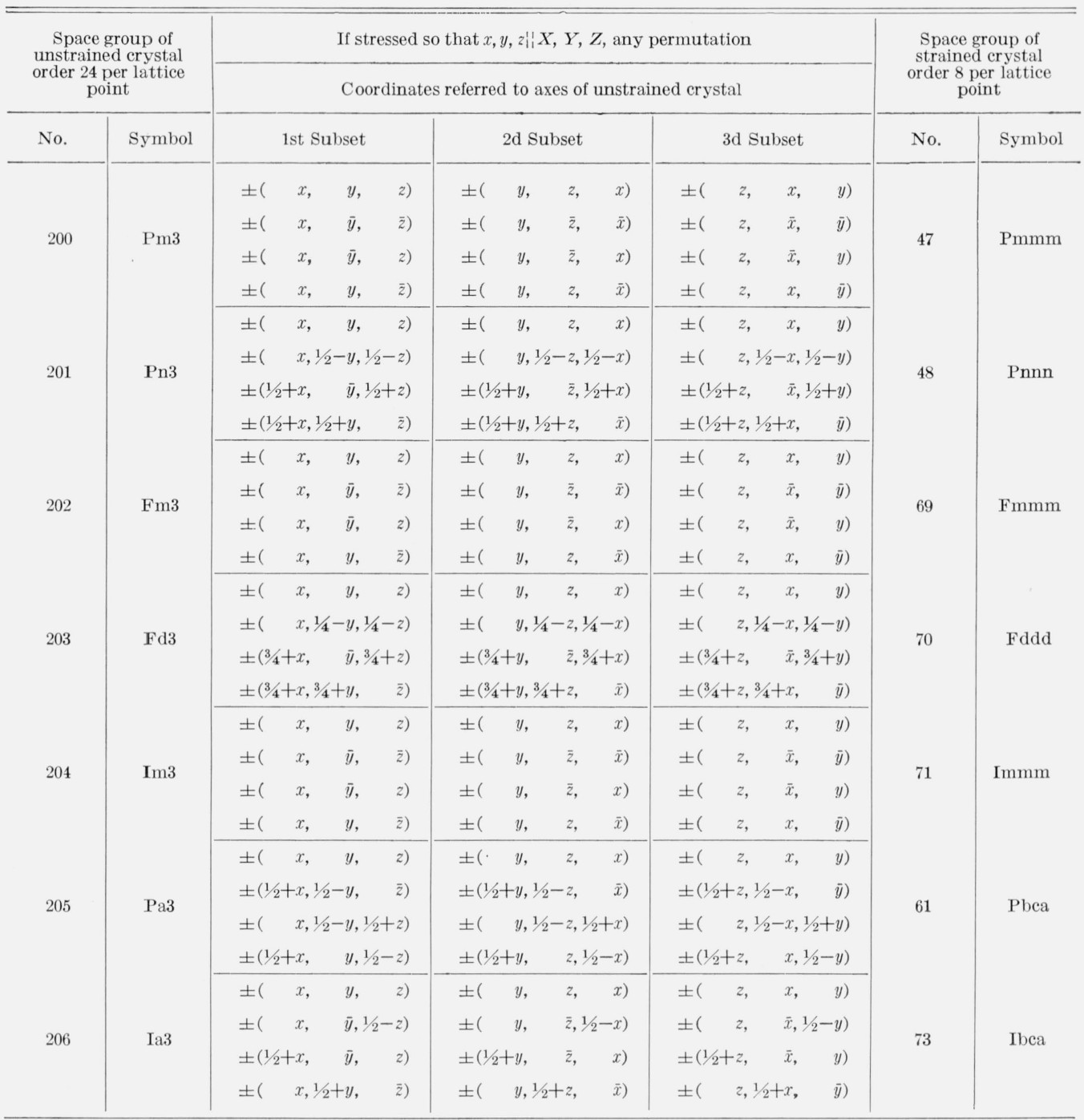

$z), \pm(x, y, \bar{z})\}$ becomes the twofold stress-induced subset $\pm(x, y, 0)$ of a special position. Similarly, $\{ \pm(x, \bar{y}, z), \pm(x, \bar{y}, \bar{z})\}$ becomes $( \pm x, \bar{y}, 0)$ so that the set of four special sites with Wyckoff symbol $p$ in Cmmm split into two subsets of special sites.

This process of specialization can be applied to obtain the stress-induced splitting of any set of special positions in any space group. Three types of behavior occur separately or at the same time:

(1) Two or more of the subsets resulting from the set making up the general position may become indistinguishable when the position is specialized. This can lead to unequal splitting such as the splitting of a set of six sites composing a special position into subsets of two and four. Unequal splitting always results in fewer subsets than splitting of a general position. The extreme case of this behavior occurs when all of the subsets resulting from the set of sites in the general positions become indistinguishable when the position is specialized. Thus many special positions do not split into subsets even though general positions always split into subsets when the space group symmetry is lowered. For unequal splitting to result from a minimum step of symmetry lowering a three-fold axis must be lost; unequal splitting can result from a compound step if a four-fold axis is lost.

(2) Sites in the same subset may become identical in clusters of two or more each. The subset then composes a special position in the stressed crystal.

(3) The number of degrees of freedom in a subset may exceed that in the original set because of loss of relevance of the restriction relative to the remaining symmetry.

An example that illustrates all three of these types of behavior at once is provided by specializing the general position in space group $\mathrm{P} \overline{3} \mathrm{ml}$, to the special position with point symmetry $\mathrm{m}$ and considering the splitting of this set of sites making up this special position into subsets under a stress that reduces $\mathrm{P} 3 \overline{\mathrm{m}} 1$ to $\mathrm{C} 2 / \mathrm{m}$. Under tensile stress along [110], the general position splits into three subsets $\{ \pm(x, y, z)$, 
TABLE 4. Reduction from m3 to $\overline{3}$

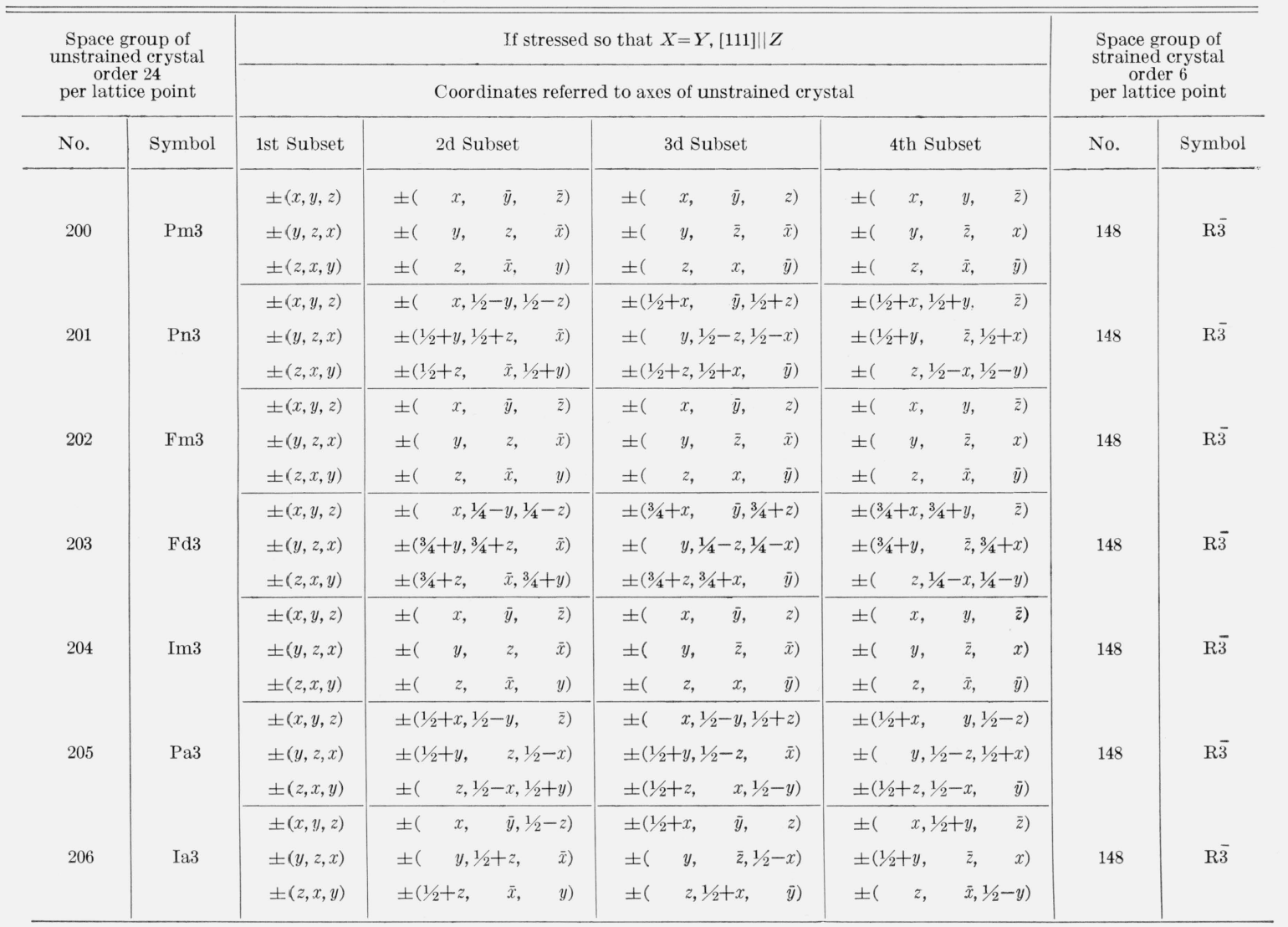

TABLE 5. Reduction from $6 / \mathrm{mmm}$ to $\mathrm{mmm}$

\begin{tabular}{|c|c|c|c|c|c|c|}
\hline \multirow{2}{*}{\multicolumn{2}{|c|}{$\begin{array}{l}\text { Space group of } \\
\text { unstrained crystal } \\
\text { order } 24 \text { per } \\
\text { lattice point }\end{array}$}} & \multirow{2}{*}{\multicolumn{3}{|c|}{$\begin{array}{l}\text { If stressed so that } z \text { and } y \| \text { any two of } X, Y, Z \\
\text { Coordinates referred to axes of unstrained crystal }\end{array}$}} & \multirow{2}{*}{\multicolumn{2}{|c|}{$\begin{array}{l}\text { Space group of } \\
\text { strained crystal } \\
\text { order } 8 \text { per } \\
\text { lattice point }\end{array}$}} \\
\hline & & & & & & \\
\hline No. & Symbol & 1st Subset & $2 d$ Subset & 3d Subset & No. & Symbol \\
\hline \multirow{3}{*}{191} & \multirow{3}{*}{$\mathrm{P} 6 / \mathrm{mmm}$} & $\pm(x, \quad y, \quad z)$ & $\pm(y, \quad x, \quad z)$ & $\pm(x-y, x$ & \multirow{3}{*}{65} & \multirow{3}{*}{$\mathrm{Cmmm}$} \\
\hline & & $\begin{array}{l} \pm(x, \quad y, \quad \bar{z}) \\
\pm(x, x-y, \quad z)\end{array}$ & $\begin{array}{l} \pm(y, \quad x, \\
\pm(y, y-x\end{array}$ & $\begin{array}{l} \pm(x-y, x, \\
\pm(x-y, \bar{y},\end{array}$ & & \\
\hline & & $\pm(x, x-y)$ & $\pm(y, y-x, \quad \bar{z})$ & $\pm(x-y, \bar{y}$ & & \\
\hline \multirow{4}{*}{192} & \multirow{3}{*}{$\mathrm{P} 6 / \mathrm{mcc}$} & $\pm(x, \quad y, \quad z)$ & $\pm(y, \quad x, 1 / 2+z)$ & $\pm(x-y, x, \quad z)$ & \multirow{3}{*}{66} & \multirow{3}{*}{ Cecm } \\
\hline & & $\begin{array}{l} \pm(x, \quad y, \quad \bar{z}) \\
\pm(x, x-y, 1 / 2+z)\end{array}$ & $\begin{array}{l} \pm(y, \quad x, 1 / 2-z) \\
\pm(y, y-x, \quad z)\end{array}$ & $\begin{array}{l} \pm(x-y, x, \quad \bar{z}) \\
\pm(x-y, \bar{y}, 1 / 2+z)\end{array}$ & & \\
\hline & & $\pm(x, x-y, 1 / 2-z)$ & $\pm(y, y-x, \quad \bar{z})$ & $\pm(x-y, \bar{y}, 1 / 2-z)$ & & \\
\hline & \multirow{3}{*}{$\mathrm{P}_{3} / \mathrm{mcm}$} & $\pm(x, \quad y, \quad z)$ & $\pm(y, \quad x, \quad z)$ & $\pm(x-y, x, 1 / 2+z)$ & \multirow{3}{*}{63} & \multirow{3}{*}{$\mathrm{Cmcm}$} \\
\hline \multirow[t]{3}{*}{193} & & $\begin{array}{l} \pm(x, \quad y, 1 / 2-z) \\
\pm(x, x-y, 1 / 2+z)\end{array}$ & $\begin{array}{l} \pm(y, \quad x, 1 / 2-z) \\
\pm(y, y-x, 1 / 2+z)\end{array}$ & $\begin{array}{ll} \pm(x-y, x, & \bar{z}) \\
\pm(x-y, \bar{y}, & z)\end{array}$ & & \\
\hline & & $\pm(x, x-y, \quad \bar{z})$ & $\pm(y, y-x, \quad \bar{z})$ & $\pm(x-y, \bar{y}, 1 / 2-z)$ & & \\
\hline & \multirow{4}{*}{$\mathrm{P}_{3} / \mathrm{mmc}$} & $\pm(x, \quad y, \quad z)$ & $\pm(y, \quad x, 1 / 2+z)$ & $\pm(x-y, x, 1 / 2+z)$ & \multirow{4}{*}{63} & \multirow{4}{*}{$\mathrm{Cmcm}$} \\
\hline \multirow{3}{*}{194} & & $\pm(x, \quad y, 1 / 2-z)$ & $\pm(y, \quad x, \quad \bar{z})$ & $\pm(x-y, x, \quad \bar{z})$ & & \\
\hline & & $\pm(x, x-y, \quad z)$ & $\pm(y, y-x, 1 / 2+z)$ & $\pm(x-y, \bar{y}, 1 / 2+z)$ & & \\
\hline & & $\pm(x, x-y, 1 / 2-z)$ & $\pm(y, y-x, \quad \bar{z})$ & $\pm(x-y, \bar{y}$ & & \\
\hline
\end{tabular}


TABle 6. Reduction from $4 / \mathrm{mmm}$ to $\mathrm{mmm}$

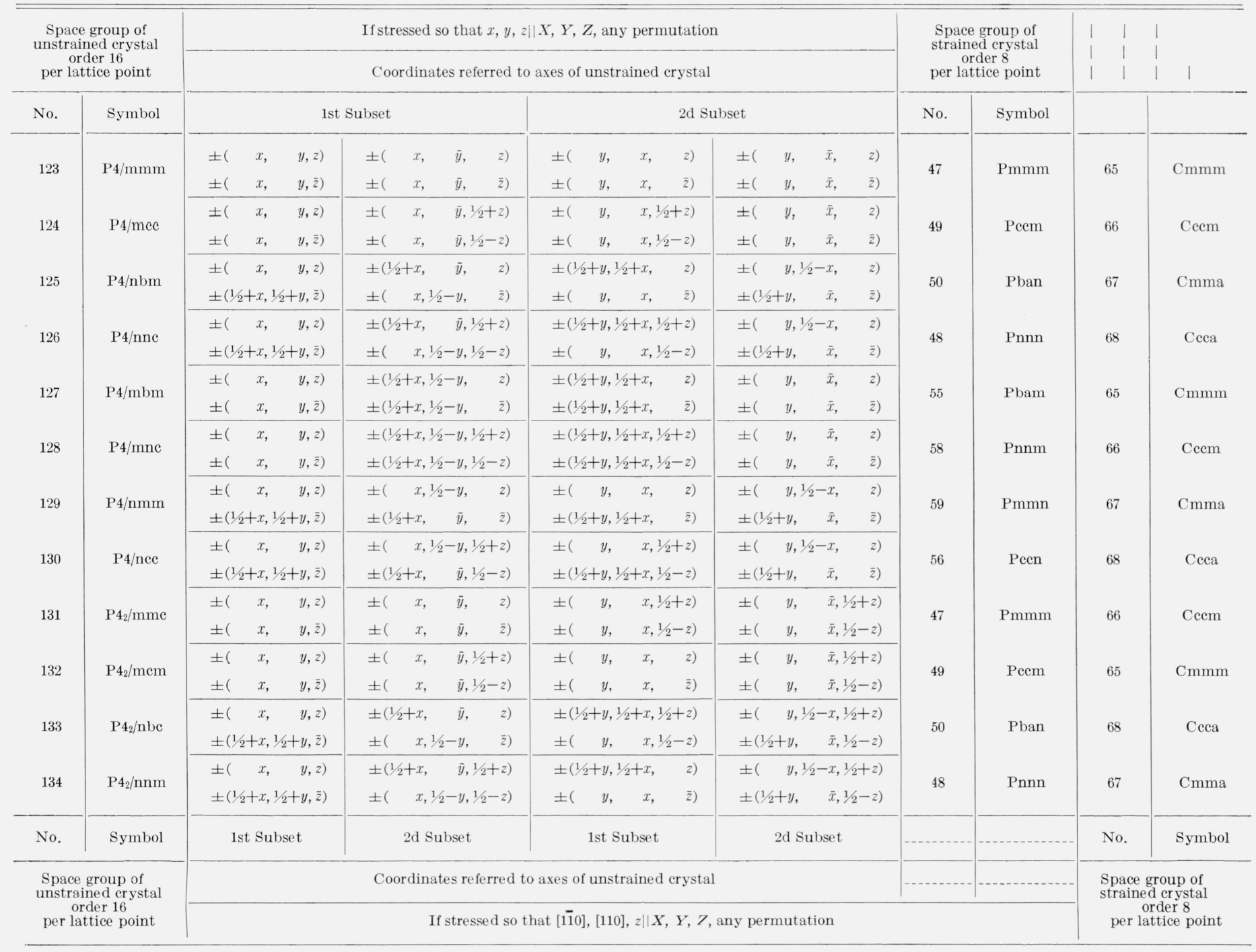


TABLE 6. Reduction from $4 / \mathrm{mmm}$ to $\mathrm{mmm}$-Continued

\begin{tabular}{|c|c|c|c|c|c|c|c|c|c|}
\hline \multirow{2}{*}{\multicolumn{2}{|c|}{$\begin{array}{l}\text { Space group of } \\
\text { unstrained crystal } \\
\text { order 16 } \\
\text { per lattice point }\end{array}$}} & \multirow{2}{*}{\multicolumn{4}{|c|}{ If stressed so that $x, y, z \| X, Y, Z$, any permutation }} & \multirow{2}{*}{\multicolumn{2}{|c|}{$\begin{array}{c}\text { Space group of } \\
\text { strained crystal } \\
\text { order } 8 \\
\text { per lattice point }\end{array}$}} & \multirow{2}{*}{$\begin{array}{ll}1 & 1 \\
1 & 1 \\
1 & 1\end{array}$} & \multirow{2}{*}{$\begin{array}{lll}1 & 1 & 1 \\
1 & 1 & 1 \\
1 & 1 & 1\end{array}$} \\
\hline & & & & & & & & & \\
\hline No. & Symbol & \multicolumn{2}{|c|}{ 1st Subset } & \multicolumn{2}{|c|}{ 2d Subset } & No. & Symbol & & \\
\hline \multirow[t]{2}{*}{135} & \multirow{2}{*}{$\mathrm{P}_{2} / \mathrm{mbc}$} & $\pm(\quad x, \quad y, z)$ & $\pm(1 / 2+x, 1 / 2-y, \quad z)$ & $\pm(1 / 2+y, 1 / 2+x, 1 / 2+z)$ & $\pm(\quad y, \quad \bar{x}, 1 / 2+z)$ & 55 & Pbam & 66 & \multirow{2}{*}{$\mathrm{Cccm}$} \\
\hline & & & $\pm(1 / 2+x, 1 / 2-y, \quad z)$ & $\pm(1 / 2+y, 1 / 2+x, 1 / 2-z)$ & $\pm(\quad y, \quad x, 1 / 2-z)$ & & & & \\
\hline 136 & $\mathrm{P}_{2} / \mathrm{mnm}$ & $\pm(\quad x, \quad y, z)$ & $\pm(1 / 2+x, 1 / 2-y, 1 / 2+z)$ & $\pm(\quad y, \quad x, \quad z)$ & $\begin{array}{l} \pm(1 / 2+y, 1 / 2-x, 1 / 2+z) \\
\pm(1 / 2+y, 1 / 2-x, 1 / 2-z)\end{array}$ & \multirow{2}{*}{$\begin{array}{l}58 \\
59\end{array}$} & Pnnm & 65 & $\mathrm{Cmmm}$ \\
\hline 137 & $\mathrm{P}_{2} / \mathrm{nmc}$ & $\begin{array}{l} \pm(\quad x, \quad y, z) \\
\pm(1 / 2+x, 1 / 2+y, \bar{z})\end{array}$ & $\begin{array}{l} \pm(\quad x, 1 / 2-y, \quad z) \\
\pm(1 / 2+x, \quad \bar{y}, \quad \bar{z})\end{array}$ & $\begin{array}{l} \pm(\quad y, \quad x, 1 / 2+z) \\
\pm(1 / 2+y, 1 / 2+x, 1 / 2-z)\end{array}$ & $\begin{array}{l} \pm(\quad y, 1 / 2-x, 1 / 2+z) \\
\pm(1 / 2+y, \quad \bar{x}, 1 / 2-z)\end{array}$ & & Pmmn & 68 & Ceca \\
\hline 138 & $\mathrm{P}_{2} / \mathrm{ncm}$ & $\begin{array}{l} \pm(\quad x, \quad y, z) \\
\pm(1 / 2+x, 1 / 2+y, \bar{z})\end{array}$ & $\begin{array}{l} \pm(\quad x, 1 / 2-y, 1 / 2+z) \\
\pm(1 / 2+x, \quad \bar{y}, 1 / 2-z)\end{array}$ & $\begin{array}{l} \pm(\quad y, \quad x, \quad z) \\
\pm(1 / 2+y, 1 / 2+x, \quad \bar{z})\end{array}$ & $\begin{array}{l} \pm(\quad y, 1 / 2-x, 1 / 2+z) \\
\pm(1 / 2+y, \quad \bar{x}, 1 / 2-z)\end{array}$ & 56 & Pcen & 67 & Cmma \\
\hline 139 & $\mathrm{I} 4 / \mathrm{mmm}$ & $\begin{array}{lll} \pm( & x, & y, z) \\
\pm( & x, & y, \bar{z})\end{array}$ & $\begin{array}{llll} \pm( & x, & \bar{y}, & z) \\
\pm( & x, & \bar{y}, & \bar{z})\end{array}$ & $\begin{array}{llll} \pm( & y, & x, & z) \\
\pm( & y, & x, & \bar{z})\end{array}$ & $\begin{array}{llll} \pm( & y, & \bar{x}, & z) \\
\pm( & y, & \bar{x}, & \bar{z}) \\
\end{array}$ & 71 & Immm & 69 & Fmmm \\
\hline 140 & $\mathrm{I} 4 / \mathrm{mem}$ & $\begin{array}{lll} \pm( & x, & y, z) \\
\pm( & x, & y, \bar{z})\end{array}$ & $\begin{array}{lll} \pm( & x, & \bar{y}, 1 / 2+z) \\
\pm( & x, & \bar{y}, 1 / 2-z)\end{array}$ & $\begin{array}{lll} \pm( & y, & x, 1 / 2+z) \\
\pm( & y, & x, 1 / 2-z)\end{array}$ & $\begin{array}{llll} \pm( & y, & \bar{x}, & z) \\
\pm( & y, & \bar{x}, & \bar{z})\end{array}$ & 72 & Ibam & 69 & Fmmm \\
\hline 141 & $14_{1} /$ amd & $\begin{array}{l} \pm(\quad x, \quad y, z) \\
\pm(\quad x, 1 / 2+y, \bar{z})\end{array}$ & $\begin{array}{lll} \pm( & x, 1 / 2-y, & z) \\
\pm( & x, \quad \bar{y}, & \bar{z})\end{array}$ & $\begin{array}{l} \pm(1 / 4+y, 1 / 4+x, 3 / 4+z) \\
\pm(1 / 4+y, 3 / 4+x, 1 / 4-z)\end{array}$ & $\begin{array}{l} \pm(1 / 4+y, 1 / 4-x, 3 / 4+z) \\
\pm(1 / 4+y, 3 / 4-x, 1 / 4-z)\end{array}$ & 74 & Imma & 70 & Fddd \\
\hline 142 & $\mathrm{I}_{1} / \mathrm{acd}$ & $\begin{array}{l} \pm(\quad x, \quad y, z) \\
\pm(\quad x, 1 / 2+y, \bar{z})\end{array}$ & $\begin{array}{ll} \pm( & x, 1 / 2-y, 1 / 2+z) \\
\pm( & x, \quad \bar{y}, 1 / 2-z)\end{array}$ & $\begin{array}{l} \pm(1 / 4+y, 1 / 4+x, 1 / 4+z) \\
\pm(1 / 4+y, 3 / 4+x, 3 / 4-z)\end{array}$ & $\begin{array}{l} \pm(1 / 4+y, 1 / 4-x, 3 / 4+z) \\
\pm(1 / 4+y, 3 / 4-x, 1 / 4-z)\end{array}$ & 73 & Ibca & 70 & Fddd \\
\hline No. & Symbol & 1st Subset & $2 d$ Subset & 1st Subset & 2d Subset & & & No. & Symbol \\
\hline $\begin{array}{r}\text { Spa } \\
\text { unstr }\end{array}$ & $\begin{array}{l}\text { group of } \\
\text { ed crystal }\end{array}$ & & Coordinates referred to & xes of unstrained crystal & & & & $\begin{array}{l}\text { Space } \\
\text { strain }\end{array}$ & $\begin{array}{l}\text { oup of } \\
\text { crystal }\end{array}$ \\
\hline per l & ice point & & If stressed so $t$ & t [11̄0], [110]. $z \| X, Y, Z$, & permutation & & & & tice point \\
\hline
\end{tabular}


TaBle 7. Reduction from $6 / m$ to $2 / m$

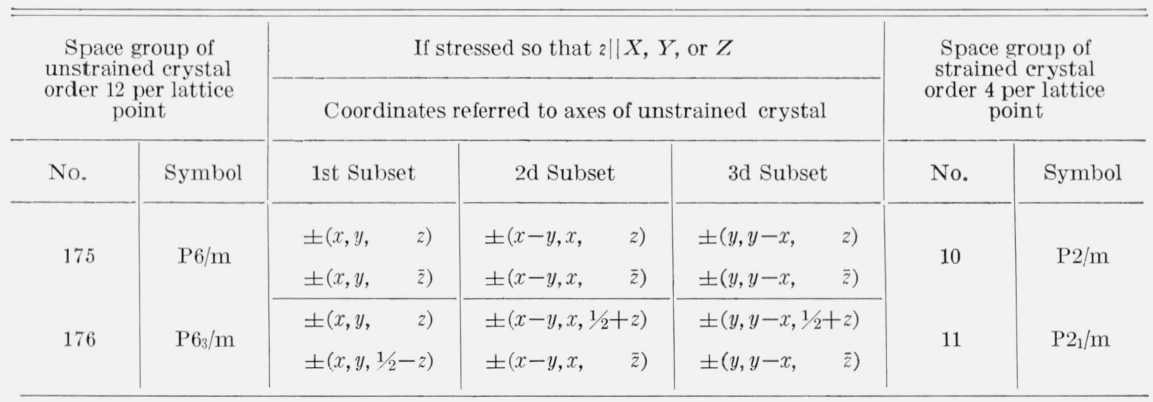

TABLE 8. Reduction from $\overline{3} m$ to $2 / m$

\begin{tabular}{|c|c|c|c|c|c|c|c|}
\hline \multicolumn{2}{|c|}{$\begin{array}{l}\text { Space group of un- } \\
\text { strained crystal order } \\
12 \text { per lattice point }\end{array}$} & \multirow[t]{2}{*}{ If stressed so that } & \multicolumn{3}{|c|}{ Coordinates referred to axes of unstrained crystal } & \multicolumn{2}{|c|}{$\begin{array}{l}\text { Space group of } \\
\text { strained crystal order } \\
4 \text { per lattice point }\end{array}$} \\
\hline No. & Symbol & & 1st Subset & $2 d$ Subset & 3d Subset & No. & Symbol \\
\hline 162 & $\mathrm{P} \overline{3} 1 \mathrm{~m}$ & {$[110] \| X, Y$, or $Z$} & $\begin{array}{l} \pm(x, y, \quad z) \\
\pm(y, x, \quad z)\end{array}$ & $\begin{array}{l} \pm(x, x-y, \quad \bar{z}) \\
\pm(x-y, \quad x, \quad \bar{z})\end{array}$ & $\begin{array}{l} \pm(\quad y, y-x, \quad \bar{z}) \\
\pm(x-y, \quad \bar{y}, \quad z)\end{array}$ & 12 & $\mathrm{C} 2 / \mathrm{m}$ \\
\hline 163 & $\overline{\mathrm{P}} \overline{3} 1 \mathrm{c}$ & {$[1 \overline{10}] \| X, Y$, or $Z$} & $\begin{array}{l} \pm(x, y, \quad z) \\
\pm(y, x, 1 / 2+z)\end{array}$ & $\begin{array}{l} \pm(x, x-y, 1 / 2-z) \\
\pm(x-y, \quad x, \quad \bar{z})\end{array}$ & $\begin{array}{l} \pm(y, y-x, \quad \bar{z}) \\
\pm(x-y, \quad \bar{y}, 1 / 2+z)\end{array}$ & 15 & $\mathrm{C} 2 / \mathrm{c}$ \\
\hline 164 & $\mathrm{P} \overline{3} \mathrm{~m} 1$ & {$[110] \| X, Y$, or $Z$} & $\begin{array}{ll} \pm(x, y, & z) \\
\pm(y, x, & \bar{z})\end{array}$ & $\begin{array}{l} \pm(x, x-y, \quad z) \\
\pm(x-y, \quad x, \quad \bar{z})\end{array}$ & $\begin{array}{l} \pm(y, y-x, \quad \bar{z}) \\
\pm(x-y, \quad \bar{y}, \quad \bar{z})\end{array}$ & 12 & $\mathrm{C} 2 / \mathrm{m}$ \\
\hline 165 & $\mathrm{P} \overrightarrow{3} \mathrm{c} 1$ & {$[110] \| X, Y$, or $Z$} & $\begin{array}{l} \pm(x, y, \quad z) \\
\pm(y, x, 1 / 2-z)\end{array}$ & $\begin{array}{l} \pm(x, x-y, 1 / 2+z) \\
\pm(x-y, \quad x, \quad \bar{z})\end{array}$ & $\begin{array}{l} \pm(y, y-x, \quad \bar{z}) \\
\pm(x-y, \quad \bar{y}, 1 / 2-z)\end{array}$ & 15 & $\mathrm{C} 2 / \mathrm{c}$ \\
\hline 166 & $\begin{array}{l}\mathrm{R} \overline{3} \mathrm{~m} \\
\text { hex. } \\
\text { axes }\end{array}$ & {$[110] \| X, Y$, or $Z$} & $\begin{array}{ll} \pm(x, y, & z) \\
\pm(y, x, & \bar{z})\end{array}$ & $\begin{array}{l} \pm(x, x-y, \quad z) \\
\pm(x-y, \quad x, \quad \bar{z})\end{array}$ & $\begin{array}{l} \pm(y, y-x, \quad \bar{z}) \\
\pm(x-y, \quad \bar{y}, \quad \bar{z})\end{array}$ & 12 & $\mathrm{C} 2 / \mathrm{m}$ \\
\hline 167 & $\begin{array}{l}\mathrm{R} \overrightarrow{3} \mathrm{c} \\
\text { hex. } \\
\text { axes }\end{array}$ & {$[110] \| X, Y$, or $Z$} & $\begin{array}{l} \pm(x, y, \quad z) \\
\pm(y, x, 1 / 2-z)\end{array}$ & $\begin{array}{l} \pm(x, x-y, 1 / 2+z) \\
\pm(x-y, \quad x, \quad \bar{z})\end{array}$ & $\begin{array}{l} \pm(y, y-x, \quad \bar{z}) \\
\pm(x-y, \quad \bar{y}, 1 / 2-z)\end{array}$ & 15 & $\mathrm{C} 2 / \mathrm{c}$ \\
\hline
\end{tabular}

TABle 9. Reduction from $4 / m$ to $2 / m$

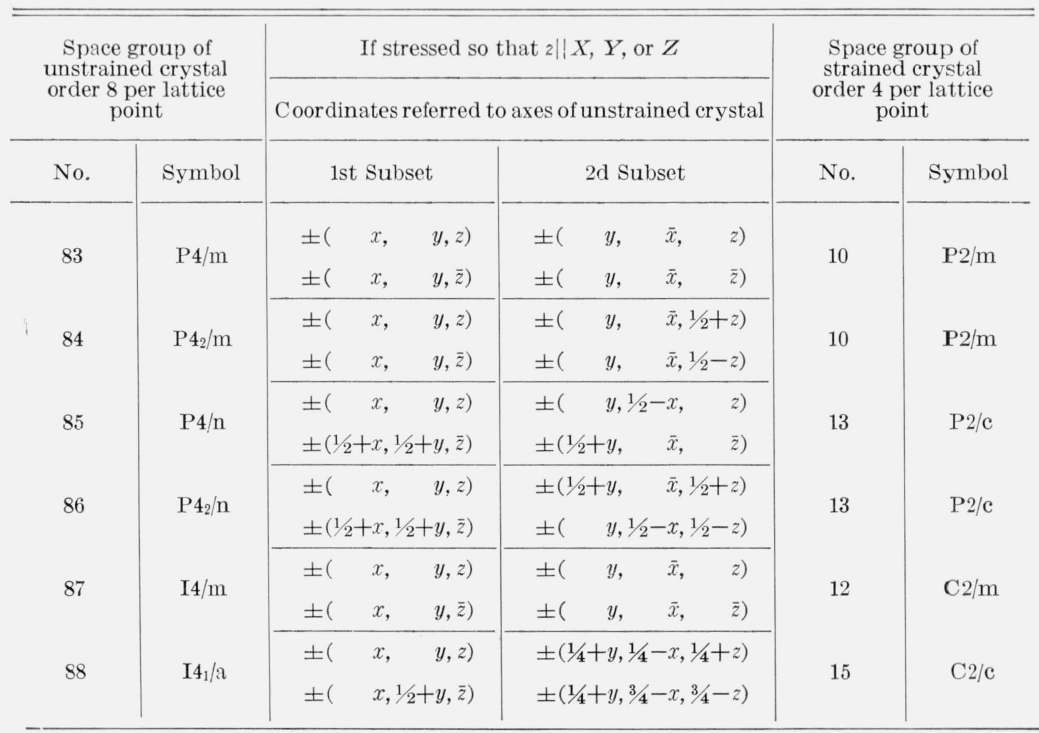


TABLE 10. Reduction from $\mathrm{mmm}$ to $2 / m$

\begin{tabular}{|c|c|c|c|c|c|c|c|c|c|c|c|c|c|}
\hline \multirow{2}{*}{\multicolumn{2}{|c|}{$\begin{array}{l}\text { Space group of } \\
\text { unstrained crystal } \\
\text { order } 8 \text { per } \\
\text { lattice point }\end{array}$}} & \multirow{2}{*}{\multicolumn{2}{|c|}{$\begin{array}{l}\text { If stressed so that } x \| X, Y \text {, or } Z \\
\begin{array}{c}\text { ordinates referred to axes of unstrained } \\
\text { crystal }\end{array}\end{array}$}} & \multirow{2}{*}{\multicolumn{2}{|c|}{$\begin{array}{l}\text { Space group of } \\
\text { strained crystal } \\
\text { order } 4 \text { per } \\
\text { lattice point }\end{array}$}} & \multirow{2}{*}{\multicolumn{2}{|c|}{$\begin{array}{c}\text { If stressed so that } y \| X, Y, \text { or } Z \\
\begin{array}{c}\text { Coordinates referred to axes of unstrained } \\
\text { crystal }\end{array}\end{array}$}} & \multirow{2}{*}{\multicolumn{2}{|c|}{$\begin{array}{l}\text { Space group of } \\
\text { strained crystal } \\
\text { order } 4 \text { per } \\
\text { lattice point }\end{array}$}} & \multirow{2}{*}{\multicolumn{2}{|c|}{$\begin{array}{c}\text { If stressed so that } z \| X, Y, \text { or } Z \\
\begin{array}{c}\text { Coordinates referred to axes of unstrained } \\
\text { crystal }\end{array}\end{array}$}} & \multirow{2}{*}{\multicolumn{2}{|c|}{$\begin{array}{l}\text { Space group of } \\
\text { strained crystal } \\
\text { order 4 per } \\
\text { lattice point }\end{array}$}} \\
\hline & & & & & & & & & & & & & \\
\hline No. & Symbol & 1st Subset & 2d Subset & No. & Symbol & 1st Subset & $2 \mathrm{~d}$ Subset & No. & Symbol & 1st Subset & $2 \mathrm{~d}$ Subset & No. & Symbol \\
\hline \multirow[t]{2}{*}{47} & \multirow[t]{2}{*}{ Pmmm } & $\pm(\quad x, \quad y, \quad z)$ & $\pm(\quad x$ & \multirow{2}{*}{10} & \multirow{3}{*}{$\begin{array}{l}\mathrm{P} 2 / \mathrm{m} \\
\mathrm{P} 2 / \mathrm{c}\end{array}$} & $\pm(\quad x, \quad y$, & $\pm(\quad x$ & \multirow{2}{*}{10} & \multirow{2}{*}{$\mathrm{P} 2 / \mathrm{m}$} & $\pm(\quad x, \quad y$, & $\pm(\quad x, \quad \bar{y}$ & \multirow{2}{*}{10} & \multirow[t]{2}{*}{$\mathrm{P} 2 / \mathrm{m}$} \\
\hline & & 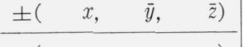 & $\pm(\quad x, \quad \bar{y}, \quad z)$ & & & $\pm(\quad x, \quad \bar{y}, \quad z)$ & $\pm(\quad x, \quad \bar{y}, \quad \bar{z})$ & & & $\pm(\quad x, \quad y$, & $\pm(\quad x, \quad \bar{y}, \quad z)$ & & \\
\hline 48 & Pnnn & $\begin{array}{l} \pm(\quad x, \quad y, \quad z) \\
\pm(\quad x, 1 / 2-y, 1 / 2-z)\end{array}$ & $\begin{array}{l} \pm(1 / 2+x, 1 / 2+y, \quad \bar{z}) \\
\pm(1 / 2+x, \quad \bar{y}, 1 / 2+z)\end{array}$ & 13 & & $\begin{array}{l} \pm(\quad x, \quad y, \quad z) \\
\pm(1 / 2+x, \quad \bar{y}, 1 / 2+z)\end{array}$ & $\begin{array}{l} \pm(1 / 2+x, 1 / 2+y, \quad \bar{z}) \\
\pm(\quad x, 1 / 2-y, 1 / 2-z)\end{array}$ & 13 & $\mathrm{P} 2 / \mathrm{c}$ & $\begin{array}{l} \pm(\quad x, \quad y, \\
\pm(1 / 2+x, 1 / 2+y\end{array}$ & $\begin{array}{l} \pm(\quad x, 1 / 2-y, 1 / 2-z) \\
\pm(1 / 2+x, \quad \bar{y}, 1 / 2+z)\end{array}$ & 13 & $\mathrm{P} 2 / \mathrm{c}$ \\
\hline 49 & Pccm & $\begin{array}{lll} \pm( & x, & y, \quad z) \\
\pm( & x, & \bar{y}, 1 / 2-z) \\
\end{array}$ & $\begin{array}{lll} \pm( & x, & y, \quad \bar{z}) \\
\pm( & x, & \bar{y}, 1 / 2+z)\end{array}$ & 13 & \multirow{2}{*}{$\begin{array}{l}\mathrm{P} 2 / \mathrm{c} \\
\mathrm{P} 2 / \mathrm{c}\end{array}$} & $\begin{array}{lll} \pm( & x, & y, \quad z) \\
\pm( & x, & \bar{y}, 1 / 2+z)\end{array}$ & $\begin{array}{lll} \pm( & x, & y, \quad \bar{z}) \\
\pm( & x, & \bar{y}, 1 / 2-z)\end{array}$ & 13 & $\mathrm{P} 2 / \mathrm{c}$ & $\begin{array}{lll} \pm( & x, & y, \\
\pm( & x, & y,\end{array}$ & $\begin{array}{lll} \pm( & x, & \bar{y}, 1 / 2-z) \\
\pm( & x, & \bar{y}, 1 / 2+z)\end{array}$ & 10 & $\mathrm{P} 2 / \mathrm{m}$ \\
\hline 50 & Pban & $\begin{array}{llr} \pm( & x, \quad y, & z) \\
\pm( & x, 1 / 2-y, & \bar{z}) \\
\end{array}$ & 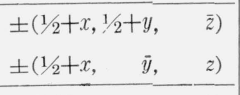 & 13 & & $\begin{array}{lrrr} \pm( & x, & y, & z) \\
\pm(1 / 2+x, & \bar{y}, & z) \\
\end{array}$ & $\begin{array}{ll} \pm(1 / 2+x, 1 / 2+y, & \bar{z}) \\
\pm(\quad x, 1 / 2-y, & \bar{z}) \\
\end{array}$ & 13 & $\mathrm{P} 2 / \mathrm{c}$ & $\begin{array}{l} \pm(\quad x, \quad y \\
\pm(1 / 2+x, 1 / 2+y\end{array}$ & $\begin{array}{l} \pm(\quad x, 1 / 2-y, \quad \bar{z}) \\
\pm(1 / 2+x, \quad \bar{y}, \quad z)\end{array}$ & 13 & $\mathrm{P} 2 / \mathbf{c}$ \\
\hline 51 & Pmma & $\begin{array}{lll} \pm(\quad x, & y, & z) \\
\pm(1 / 2+x, & \bar{y}, & \bar{z})\end{array}$ & $\begin{array}{lll} \pm(1 / 2+x, & y, & \bar{z}) \\
\pm(\quad x, & \bar{y}, & z)\end{array}$ & 11 & $\mathrm{P} 2_{1} / \mathrm{m}$ & $\begin{array}{llll} \pm( & x, & y, & z) \\
\pm( & x, & \bar{y}, & z)\end{array}$ & $\begin{array}{lll} \pm(1 / 2+x, & y, & \bar{z}) \\
\pm(1 / 2+x, & \bar{y}, & \bar{z}) \\
\end{array}$ & 10 & $\mathrm{P} 2 / \mathrm{m}$ & $\begin{array}{l} \pm(\quad x, \quad y, \\
\pm(1 / 2+x, \quad y,\end{array}$ & $\begin{array}{lll} \pm(1 / 2+x, & \bar{y}, & \bar{z}) \\
\pm(\quad x, & \bar{y}, & z)\end{array}$ & 13 & $\mathrm{P} 2 / \mathrm{c}$ \\
\hline 52 & Pnna & $\begin{array}{l} \pm(x, \quad y, \quad z) \\
\pm(\quad x, 1 / 2-y, 1 / 2-z)\end{array}$ & $\begin{array}{l} \pm(1 / 2+x, \quad y, \quad \bar{z}) \\
\pm(1 / 2+x, 1 / 2-y, 1 / 2+z)\end{array}$ & 13 & $\mathrm{P} 2 / \mathrm{c}$ & $\begin{array}{l} \pm(\quad x, \quad y, \quad z) \\
\pm(1 / 2+x, 1 / 2-y, 1 / 2+z)\end{array}$ & $\begin{array}{l} \pm(1 / 2+x, \quad y, \quad \bar{z}) \\
\pm(\quad x, 1 / 2-y, 1 / 2-z)\end{array}$ & 14 & $\mathrm{P} 2_{1} / \mathrm{c}$ & $\begin{array}{lrrr} \pm(\quad x, & y, & z) \\
\pm(1 / 2+x, & y, & \bar{z})\end{array}$ & $\begin{array}{l} \pm(\quad x, 1 / 2-y, 1 / 2-z) \\
\pm(1 / 2+x, 1 / 2-y, 1 / 2+z)\end{array}$ & 13 & $\mathrm{P} 2 / \mathrm{c}$ \\
\hline 53 & Pmna & $\begin{array}{llll} \pm( & x, & y, & z) \\
\pm( & x, & \bar{y}, & \bar{z}) \\
\end{array}$ & $\begin{array}{ll} \pm(1 / 2+x, & y, 1 / 2-z) \\
\pm(1 / 2+x, & \bar{y}, 1 / 2+z)\end{array}$ & 10 & $\mathrm{P} 2 / \mathrm{m}$ & $\begin{array}{ll} \pm(\quad x, & y, \quad z) \\
\pm(1 / 2+x, & \bar{y}, 1 / 2+z)\end{array}$ & $\begin{array}{lll} \pm(1 / 2+x, & y, 1 / 2-z) \\
\pm(\quad x, & \bar{y}, \quad \bar{z})\end{array}$ & 13 & $\mathrm{P} 2 / \mathrm{c}$ & $\begin{array}{l} \pm(\quad x, \quad y, \quad z) \\
\pm(1 / 2+x, \quad y, 1 / 2-z)\end{array}$ & $\begin{array}{ll} \pm(\quad x, & \bar{y}, \quad \bar{z}) \\
\pm(1 / 2+x, & \bar{y}, 1 / 2+z)\end{array}$ & 14 & $\mathrm{P} 2_{1} / \mathrm{c}$ \\
\hline 54 & Peca & 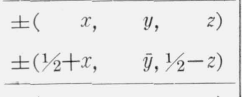 & $\begin{array}{ll} \pm(1 / 2+x, & y, \quad \bar{z}) \\
\pm(\quad x, & \bar{y}, 1 / 2+z)\end{array}$ & 14 & $\mathrm{P} 2_{1} / \mathrm{c}$ & $\begin{array}{lllr} \pm( & x, & y, \quad z) \\
\pm( & x, & \bar{y}, 1 / 2+z) \\
\end{array}$ & $\begin{array}{llr} \pm(1 / 2+x, & y, \quad \bar{z}) \\
\pm(1 / 2+x, & \bar{y}, 1 / 2-z) \\
\end{array}$ & 13 & $\mathrm{P} 2 / \mathrm{c}$ & $\begin{array}{lrrr} \pm(\quad x, & y, & z) \\
\pm(1 / 2+x, & y, & \bar{z}) \\
\end{array}$ & $\begin{array}{ll} \pm(1 / 2+x, & \bar{y}, 1 / 2-z) \\
\pm(\quad x, & \bar{y}, 1 / 2+z)\end{array}$ & 13 & $\mathrm{P} 2 / \mathrm{c}$ \\
\hline 55 & Pbam & 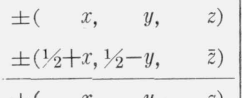 & $\begin{array}{l} \pm(\quad x, \quad y, \quad \bar{z}) \\
\pm(1 / 2+x, 1 / 2-y,\end{array}$ & 14 & $\mathrm{P} 2_{1} / \mathrm{c}$ & $\begin{array}{l} \pm(x, \quad y, \quad z) \\
\pm(1 / 2+x, 1 / 2-y,\end{array}$ & 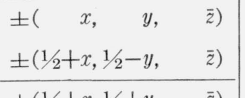 & 14 & $\mathrm{P} 2_{1} / \mathrm{c}$ & $\begin{array}{llll} \pm( & x, & y, & z) \\
\pm( & x, & y, & \bar{z}) \\
\end{array}$ & $\begin{array}{ll} \pm(1 / 2+x, 1 / 2-y, & \bar{z}) \\
\pm(1 / 2+x, 1 / 2-y, & z)\end{array}$ & 10 & $\mathrm{P} 2 / \mathrm{m}$ \\
\hline 56 & Pecn & $\begin{array}{lllr} \pm( & x, & y, & z) \\
\pm(1 / 2+x, & \bar{y}, 1 / 2-z) \\
+( & x\end{array}$ & $\begin{array}{l} \pm(1 / 2+x, 1 / 2+y, \quad \bar{z}) \\
\pm(\quad x, 1 / 2-y, 1 / 2+z)\end{array}$ & 14 & $\mathrm{P} 2_{1} / \mathrm{c}$ & $\begin{array}{ll} \pm( & x, \quad y, \quad z) \\
\pm( & x, 1 / 2-y, 1 / 2+z) \\
\end{array}$ & $\begin{array}{l} \pm(1 / 2+x, 1 / 2+y, \quad \bar{z}) \\
\pm(1 / 2+x, \quad \bar{y}, 1 / 2-z)\end{array}$ & 14 & $\mathrm{P} 2_{1} / \mathrm{c}$ & 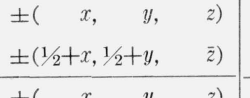 & $\begin{array}{l} \pm(1 / 2+x, \quad \bar{y}, 1 / 2-z) \\
\pm(\quad x, 1 / 2-y, 1 / 2+z)\end{array}$ & 13 & $\mathrm{P} 2 / \mathrm{c}$ \\
\hline 57 & $\mathrm{Pbcm}$ & 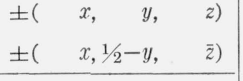 & $\begin{array}{ll} \pm( & x, \quad y, 1 / 2-z) \\
\pm( & x, 1 / 2-y, 1 / 2+z)\end{array}$ & 13 & $\mathrm{P} 2 / \mathrm{c}$ & $\begin{array}{ll} \pm( & x, \quad y, \quad z) \\
\pm( & x, 1 / 2-y, 1 / 2+z)\end{array}$ & $\begin{array}{ll} \pm( & x, \quad y, 1 / 2-z) \\
\pm( & x, 1 / 2-y, \quad \bar{z})\end{array}$ & 14 & $\mathrm{P} 2_{1} / \mathrm{c}$ & $\begin{array}{lllr} \pm( & x, & y, \quad z) \\
\pm( & x, & y, 1 / 2-z)\end{array}$ & $\begin{array}{ll} \pm( & x, 1 / 2-y, \quad \bar{z}) \\
\pm( & x, 1 / 2-y, 1 / 2+z)\end{array}$ & 11 & $\mathrm{P}_{1} / \mathrm{m}$ \\
\hline 58 & Pnnm & $\begin{array}{l} \pm(\quad x, \quad y, \quad z) \\
\pm(1 / 2+x, 1 / 2-y, 1 / 2-z)\end{array}$ & $\begin{array}{l} \pm(\quad x, \quad y, \quad \bar{z}) \\
\pm(1 / 2+x, 1 / 2-y, 1 / 2+z)\end{array}$ & 14 & $\mathrm{P} 2_{1} / \mathrm{c}$ & $\begin{array}{l} \pm(\quad x, \quad y, \quad z) \\
\pm(1 / 2+x, 1 / 2-y, 1 / 2+z)\end{array}$ & $\begin{array}{l} \pm(x, \quad y, \quad \bar{z}) \\
\pm(1 / 2+x, 1 / 2-y, 1 / 2-z)\end{array}$ & 14 & $\mathrm{P} 2_{1} / \mathrm{c}$ & $\begin{array}{llll} \pm( & x, & y, & z) \\
\pm( & x, & y, & \bar{z}) \\
\end{array}$ & $\begin{array}{l} \pm(1 / 2+x, 1 / 2-y, 1 / 2-z) \\
\pm(1 / 2+x, 1 / 2-y, 1 / 2+z)\end{array}$ & 10 & $\mathrm{P} 2 / \mathrm{m}$ \\
\hline 59 & Pmmn & $\begin{array}{l} \pm(\quad x, \quad y, \\
\pm(1 / 2+x, \quad \bar{y},\end{array}$ & 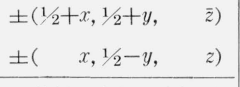 & 11 & $\mathrm{P} 22_{1} / \mathrm{m}$ & $\begin{array}{l}x, \quad y, \quad z) \\
x, 1 / 2-y, \quad z) \\
\end{array}$ & $\begin{array}{lll} \pm(1 / 2+x, 1 / 2+y, & \bar{z}) \\
\pm(1 / 2+x, & \bar{y}, & \bar{z}) \\
\end{array}$ & 11 & $\mathrm{P} 2{ }_{1} / \mathrm{m}$ & $\begin{array}{l} \pm(\quad x, \quad y, \quad z) \\
\pm(1 / 2+x, 1 / 2+y, \quad \bar{z})\end{array}$ & $\begin{array}{l} \pm(1 / 2+x, \quad \bar{y}, \quad \bar{z}) \\
\pm(\quad x, 1 / 2-y, \quad z) \\
\end{array}$ & 13 & $\mathrm{P} 2 / \mathrm{c}$ \\
\hline 60 & Pbcn & $\begin{array}{l} \pm(\quad x, \quad y \\
\pm(1 / 2+x, 1 / 2-y,\end{array}$ & $\begin{array}{l} \pm(1 / 2+x, 1 / 2+y, 1 / 2-z) \\
\pm(\quad x, \quad \bar{y}, 1 / 2+z)\end{array}$ & 14 & $\mathrm{P} 2_{1} / \mathrm{c}$ & $\begin{array}{lllr} \pm( & x, & y, \quad z) \\
\pm( & x, & \bar{y}, 1 / 2+z) \\
\end{array}$ & $\begin{array}{l} \pm(1 / 2+x, 1 / 2+y, 1 / 2-z) \\
\pm(1 / 2+x, 1 / 2-y, \quad \bar{z}) \\
\end{array}$ & 13 & $\mathrm{P} 2 / \mathrm{c}$ & $\begin{array}{l} \pm(\quad x, \quad y, \quad z) \\
\pm(1 / 2+x, 1 / 2+y, 1 / 2-z)\end{array}$ & $\begin{array}{l} \pm(1 / 2+x, 1 / 2-y, \quad \bar{z}) \\
\pm(\quad x, \quad \bar{y}, 1 / 2+z)\end{array}$ & 14 & $\mathrm{P} 2_{1} / \mathrm{c}$ \\
\hline
\end{tabular}




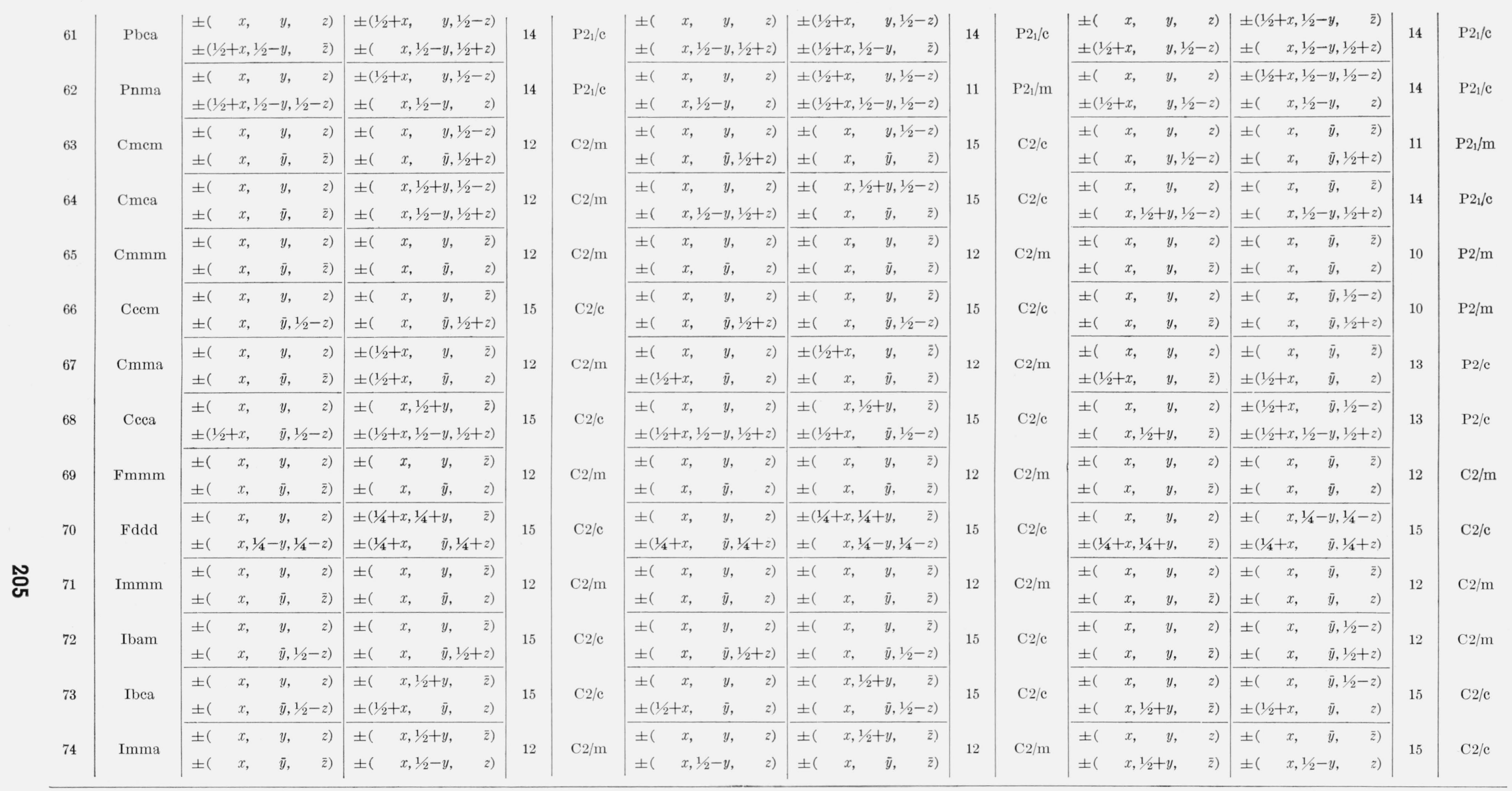


TABLE 11. Reduction from $\overline{3}$ to $\overline{1}$

\begin{tabular}{|c|c|c|c|c|c|c|}
\hline \multirow{2}{*}{\multicolumn{2}{|c|}{$\begin{array}{l}\text { Space group of } \\
\text { unstrained crystal } \\
\text { order } 6 \\
\text { per lattice point }\end{array}$}} & \multirow{2}{*}{\multicolumn{3}{|c|}{ Coordinates referred to axes of unstrained crystal }} & \multirow{2}{*}{\multicolumn{2}{|c|}{$\begin{array}{l}\text { Space group of } \\
\text { strained crystal } \\
\text { order } 2 \\
\text { per lattice point }\end{array}$}} \\
\hline & & & & & & \\
\hline No. & Symbol & 1st Subset & $2 d$ Subset & 3d Subset & No. & Symbol \\
\hline 147 & $\mathrm{P} \overline{3}$ & $\pm(x, y, z)$ & $\pm(y, y-x, \bar{z})$ & $\pm(x-y, x, \bar{z})$ & 2 & $\mathrm{P} \overline{1}$ \\
\hline 148 & $\begin{array}{c}\mathrm{R} \overline{3} \\
\text { hex. axes }\end{array}$ & $\pm(x, y, z)$ & $\pm(y, y-x, \bar{z})$ & $\pm(x-y, x, \bar{z})$ & 2 & $\mathrm{P} \overline{1}$ \\
\hline
\end{tabular}

TABLE 12 . Reduction from $2 / m$ to $\overline{1}$

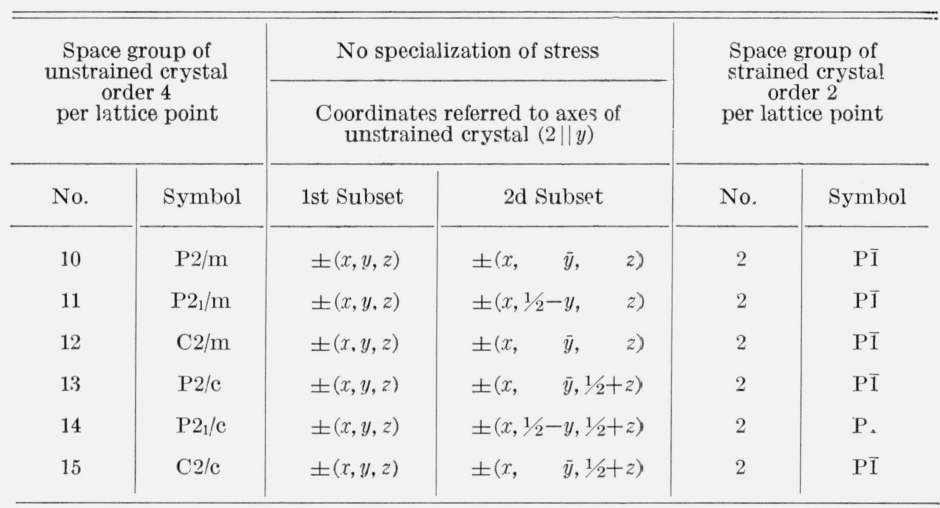

TABLE 13. Most general stress consistent with a reduction of a centrosymmetric point group to any one of its stress-induced subgroups (hexagonal axes used for trigonal groups)

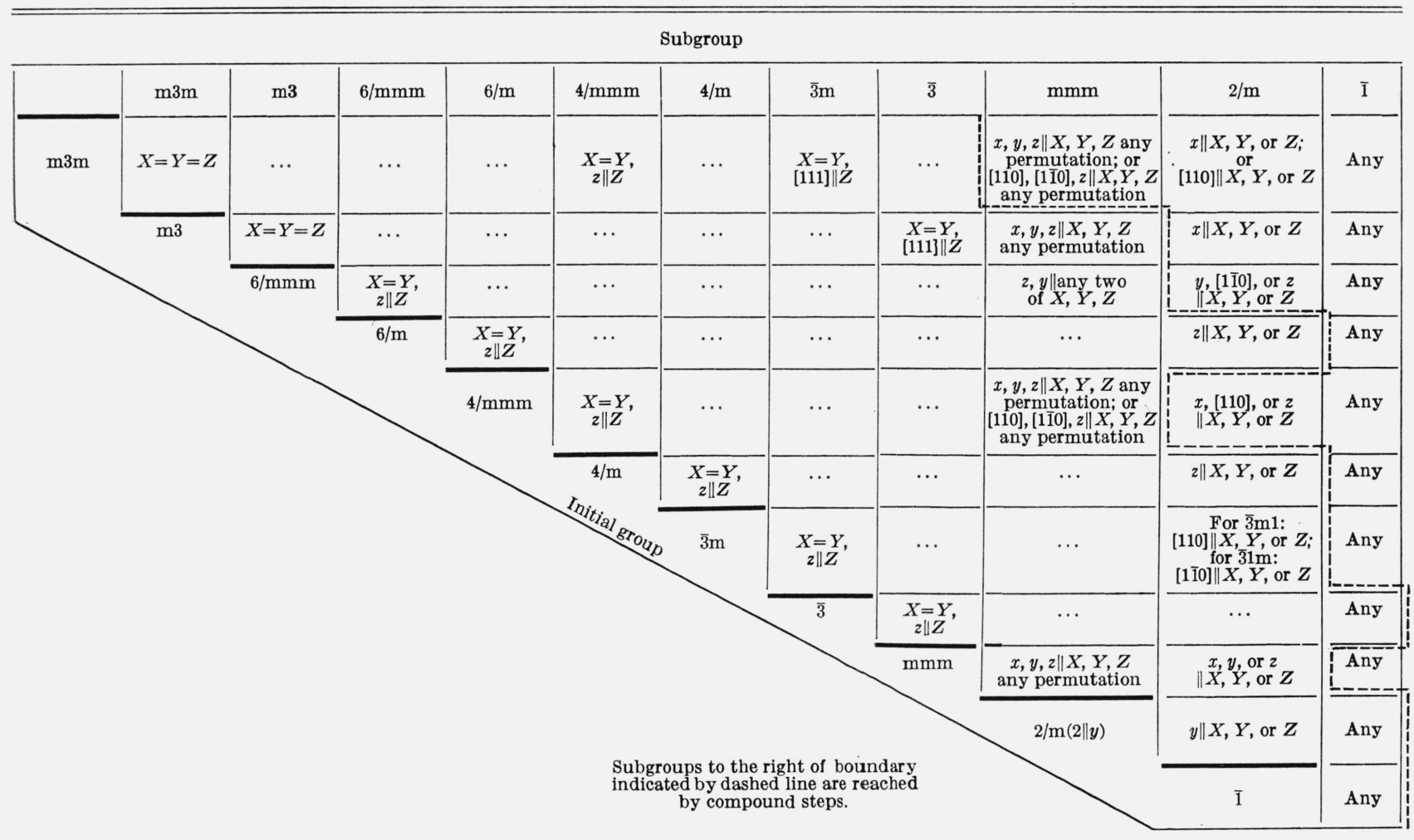


$\pm(y, x, \bar{z})\}, \quad\{ \pm(x, x-y, z), \pm(x-y, x, \bar{z})\}$, and $\{ \pm(y, y-x, \bar{z}), \pm(x-y, \bar{y}, \bar{z})\}$ as the tables show. The special position with point symmetry $\mathrm{m}$ in $\mathrm{P} \overline{3} \mathrm{~m} 1$ is designated by Wyckoff symbol $i$ in the International Tables and can be generated from the point $(x, x, z)$. We then take $y=x$ throughout the three subsets and examine the resulting types of behavior. Type 1 is shown by subsets two and three because each site in one becomes identical to a site in the other. 'The resulting subset is $\{ \pm(x, 2 x, z)$, $\pm(2 x$, $x, z)\}$. Type 2 is shown by the first subset in which pairs of sites become identical resulting in the subset $\{ \pm(x, \bar{x} z)\}$. The two sites in this last subset lie on the mirror plane remaining in $\mathrm{C} 2 / \mathrm{m}$ and so constitute a set of special positions in the stressed crystal. In contrast, the sites in the subset resulting from the original second and third subsets do not lie on any symmetry element of $\mathrm{C} 2 / \mathrm{m}$ so the site $(x, 2 x$, z) should be written $(x, y, z)$ because there is no longer any symmetry constraining $y$ to equal $2 x$ and the subset becomes $\{ \pm(x, y, z), \pm(y, x, z)\}$ which composes a general position in $\mathrm{C} 2 / \mathrm{m}$. In this example of type 3 behavior, four of the sites of a special position with two degrees of freedom map onto the general position and so gain an additional degree of freedom. Mapping onto a general position occurs if and only if the number in a subset equals the order of the point group of the stressed crystal.

The authors are indebted to Dr. J. D. H. Donnay for having read this paper in manuscript and made several pertinent suggestions.

\section{References}

[1] B. S. Berry, Review of internal friction due to point defects, Acta. Met. 10, 271 (1962).

[2] J. B. Wachtman, Jr., and H. S. Peiser, Symmetry conditions for internal friction caused by jumping of point defects in crystals, Appl. Phys. Letters 1, 20 (1962).

[3] A. S. Nowick and W. R. Heller, Anelasticity and stress induced ordering of point defects in crystals, Adv. in Phys. (Phil. Mag. supplement) 12, 251 (1963).

[4] J. B. Wachtman, Jr., H. S. Peiser, and E. P. Levine, Symmetry splitting of equivalent sites in oxide crystals and related mechanical effects, J. Res. NBS $6 \mathbf{6 A}$, 281 (1963).

[5] A. S. Nowick and W. R. Heller, Criteria for occurrence of internal friction due to point defects in crystals, Bull. Am. Phys. Soc. 8, 236 (1963).

[6] H. S. Peiser, J. B. Wachtman, Jr., and R. W. Dickson, Reduction of space groups to subgroups by homogeneous strain, J. Res. NBS 67A, 395 (1963).

[7] J. F. Nye, Physical properties of crystals, Oxford University Press (1957).

[8] A. D. Franklin, Relaxation modes for trapped crystal point defects, J. Res. NBS 67A, 291 (1963).

[9] International Tables for X-ray Crystallography, Vol. I. Edited by N. F. M. Henry and K. Lonsdale, Kynoch Press (Birmingham, 1952).

[10] Internationale Tabellen zur Bestimmung von Kristallstrukturen, Erster Band, Gruppentheoretische Tafeln xii +452 pp. Gebrueder Borntraeger, Berlin (1935), (Reprinted with corrigenda by Edwards Brothers. Inc., Ann Arbor, Mich., 1944.)

(Paper 69A2-340) 


\section{Publications of the National Bureau of Standards*}

\section{Selected Abstracts}

Construction of a Michelson interferometer for Fourier spectroscopy, H. N. Rundle, J. Res. NBS 69C (Engr. and Instr.), No. 1 (Jan.-Mar. 1964).

A brief outline is presented of the theory of the use of a double beam interferometer as applied to Fourier spectroscopy. A Michelson interferometer is described which is suitable for use in Fourier spectroscopy in the 1 to 10 micron region. The instrument can be used at any resolving power up to about $10^{5}$.

The National Bureau of Standards gas thermometer II. Measurement of capacitance to a grounded surface with a transformer ratio-arm bridge, L. A. Guildner and R. E. Edsinger, J. Res. NBS 69C (Eng. and Instr.), No. 1 (Jan.Mar. 1964).

A modification of the conventional transformer ratio-arm capacitance bridge was made to measure the values of three lead capacitors having one of the plates grounded. With this ground point it is necessary to use tri-axial cable with solid shields. A troublesome capacitance coupling between the primary and secondary shields of the transformer was neutralized by means whice still enable one to attain the extremely high precision and accuracy characteristic of this type of bridge. The modified bridge was developed initially for highly accurate determinations of the locations of the grounded mercury menisei of the National Bureau of Standards precision manometer. Other applications are readily thought of and one interesting example is presented.

An adiabatic calorimeter for the range, 10 to $360 \mathrm{~K}$., K. F Sterrett, D. H. Blackburn, A. B. Bestul, S. S. Chang, and J. Horman, J. Res. NBS 69C (Eng. and Instr.), No. 1, (Jan.Mar. 1965).

A low-temperature adiabatic calorimeter and cryostat assembly is described for measuring heat capacities in the temperature range 10 to $360^{\circ} \mathrm{K}$. A combination of dewar and tank system is used as refrigerant containers. The temperature of the aaiabatic shield is automatically controlled to within a millidegree of that of the calorimeter vessel. This apparatus offers iacilities for rapid cooling to about $55^{\circ} \mathrm{K}$ and long term adiabatic control for conditioning of a sample and, thus, is especially suitable for measurements on glass, where long equilibration time is sometimes involved.

Data on the empty calorimeter vessel and on the Calorimetry Conference standard sample of synthetic sapphire are presented as a measure of the precision and the accuracy of the apparatus.

Effect of electron collisions on the formulas of magneto ionic theory, K. G. Budden, Radio Sci. J. Res. NBS/USNCURSI, Vol. 69D, No. \& (Feb. 1965).

In the standard simple treatment of magneto ionic theory the effect of collisions is allowed for by assuming that an electron experiences a retarding force proportional to its velocity, and the two complex refractive indices of an ionized medium are then given by the Appleton-Hartree formula. Experiments have shown, however, that the collision frequency is approximately proportional to the square of the electron's velocity, and proper allowance for this, using the Boltzmann equation, leads to a modification of the Appleton-Hartree formula which was given by Sen and Wyller. In this tutorial paper the same modified formula is derived, but by a different method which, it is hoped, can be followed by readers not intimately familiar with the previous literature. Some numerical results are presented in which the modified and unmodified formulae are compared. It is concluded that the standard Appleton-Hartree formula can be used without modification for nearly all radio propagation problems in the ionosphere, provided that the correct effective value of the collision frequency is used. The modifications may be important, however, in the theory of wave interaction and for waves of very low frequency whose wave normals are perpendicular to the earth's magnetic field.

Discussion of basic equations with source terms in compressive plasmas, Kun-Mu Chen, Radio Sci. J. Res. NBS USNC-URSI, Vol. 69D, No. 2 (Feb. 1965).

It is shown that the same set of basic equations with source terms can and should be applied to both a compressive and an incompressive isotropic plasma. In a compressive plasma, an electric source can excite an electroacoustic wave. In an incompressive plasma, however, this electroacoustic wave reduces to an accumulation of charges shielding the electric source. The physical meaning of the dielectric constant of an incompressive plasma is discussed and the physical model of an incompressive plasma is re-examined.

The electrical properties of aluminum for cryogenic electromagnets, R. J. Corruccini, NBS Tech. Note 218 (Aug. 30,1964), 30 cents.

The published data for the ideal resistivity and the magnetoresistivity of aluminum have been correlated. It is shown that both properties can be calculated for the limited ranges of temperature and purity that are of importance for aluminum cryogenic electromagnets from the residual resistivity ratio alone. Empirical functions are given for these properties, and sample calculations are given of the figure of merit for a particular aluminum solenoid relative to the same solenoid using water-cooled copper.

Demagnetizing factors for oblate spheroids used in ferrimagnetic resonance measurements, L. B. Schmidt, W. E. Case, and R. D. Harrington, NBS Tech. Note 221 (Sept. 4, 1964), 20 cents.

Demagnetizing factors for oblate spheroids magnetized along the short axis are given for aspect ratios from 25.0 to 35.0 in increments of 0.1 , from 35.0 to 55.0 in increments of 0.2 , from 55.0 to 80.0 in increments of 0.5 , from 80.0 to 129.0 in increments of 1.0. The values of all demagnetizing factors given in the tables have been rounded off to 6 decimal places and are accurate to \pm 5 units in the seventh place. The tables are presented in a form convenient for use in ferrimagnetic resonance measurements on disk shaped samples. A brief discussion of the effect of accuracy of demagnetizing factors on measurements of this type is included.

Survey of magnetic thin film materials, G. W. Reimherr, NBS Tech. Note 247 (Sept. 25, 1964), 40 cents.

This survey lists the materials reportedly made as a magnetic thin film, along with some of their properties and potential applications. Research activity using the less-frequently mentioned magnetic film materials is noted. About 200 references are cited.

Electronic energy bands in strontium titanate, A. H. Kahn and A. J. Leyendecker, Phys. Rev. 135, No. 5A, 1321-1225 (Aug. 31, 196 4).

A theoretical examination of the electronic energy bands of cubic strontium titanate has been performed by application of the LCAO (linear combination of atomic orbitals) method. Diagonal energies were determined from ionization potentials 
and crystalline fields based on a point charge model for the ions. The ionic charges were adjusted to give agreement with the observed energy gap. Overlap integrals were estimated from free ion wave functions. For the crystals studied, the calculations led to filled valence bands derived primarily from oxygen $2 p$ orbitals and empty conduction bands derived predominantly from titanium $3 d$ orbitals. In cubic strontium and barium titanates, there are six lowest conduction band ellipsoids lying along (100) directions of $k$-space with minima probably at the edges of the Brillouin zone. The longitudinal mass is about $20-50 \mathrm{~m}_{\mathrm{o}}$ and the transverse mass about $1 \mathrm{~m}_{\mathrm{o}}$. Spin-orbit splitting removes degeneracy at $k=0$ and leads to additional conduction bands several hundredths of an ev above the lowest conduction band.

Comparison is made with experimental data on conductivity, Hall effect, thermoelectric power, reflectivity, and soft X-ray emission. Results are in substantial agreement with experiment.

Experiments on self-ionizing shock waves in a magnetic field, Y. Nakagawa and K. B. Earnshaw, Book, Comptes Rendus de la $V I \mathrm{e}$ Conference Internationale sur les Phenomenes D'Ionisation dans les Gaz, ed. P. Hubert and E. CremieuAlcan, IV, 303-309 (S.E.R.M.A., Paris, France, 1963).

The structure of a self-ionizing shock wave in He of $50 \mu \mathrm{Hg}$ has been studied experimentally. The shock wave was produced by means of an electric discbarge and part of the shock tube was placed in a transverse magnetic field. By varving the strength of this magnetic field, it was possible to study the variation of the structure of the shock as well as the state of ionization behind the shock in the range of Mach number from 40 to 99.5 and of Magnetic Mach number (shock velocity/Alfvén velocity 0.18 to 20 . It was found that ionization behind the shock wave can be best explained in terms of streams of electrons and ions produced by the discharge.

Refractivities of the rare earth oxides, E. M. Levin, (Proc. Third Rare Earth Research Conf., Apr. 2-24, 1963), Book, Rare Earth Research, Chapter on Magnetic and Ontical Studies, pp. 339-350 (Gordon and Breach, Science Publ., Inc., New York, N.Y., May 1964).

Index of refraction and density data obtained for a number of rare earth borates $\left(\mathrm{ABO}_{3}\right)$ and aluminates and gallates $\left(\mathrm{A}_{2} \mathrm{~B}_{2} \mathrm{O}_{12}\right)$ permitted the calculation of the specific refractivities (by the Gladstone and Dale formula) of the rare earth sesquioxides. This was done using the additive rule between the products of the specific refractivities and the weight fractions of the constituent oxides. The specific refractivity values decreased with decreasing ionic radius of the cation, ranging from 0.148 for $\mathrm{La}_{2} \mathrm{O}_{3}$ to 0.107 for $\mathrm{Dy}_{2} \mathrm{O}_{3}$ to 0.090 for $\mathrm{Lu}_{2} \mathrm{O}_{3}$, and include the lowest values ever reported for oxides. Specific refractivities and molar refractivities (LorentzLorenz formula) were calculated also from data on crystal fragments of the cubic rare earth oxides, obtained from two sources. Sperific refractivities decreased from a value of 0.120 for $\mathrm{Dy}_{2} \mathrm{O}_{3}$ to 0.099 for $\mathrm{Lu}_{2} \mathrm{O}_{3}$, and were 10 to $13 \%$ higher than those calculated from the binary oxide compounds. Molar refractivities (Lorentz-Lorenz) of the pure oxides varied from 22.5 for $\mathrm{Dy}_{2} \mathrm{O}_{3}$ to 20.2 for $\mathrm{Lu}_{2} \mathrm{O}_{3}$ and were in good agreement with reported data.

Vibration-rotation bands of deuterium cyanide and hydrogen cyanide, A. G. Maki, E. K. Plyler, and R. Thibault, J. Opt. Soc. Am. 54, No. 7, 869-876 (July 1964).

Six vibration-rotation bands of DCN between 2 and 6 microns have been analyzed. All the rotational constants have been obtained through the terms quadratic in vibrational quantum numbers. Two quadratic and seven cubic vibrational anharmonic constants have been obtained explicitly. Seven combinations of quadratic and cubic vibrational constants have also been obtained. Data are given on the analysis of the two parallel fundamental bands of $\mathrm{DC}^{13} \mathrm{~N}$, as well as the $\nu_{3}$ bands of $\mathrm{DCN}^{15}, \mathrm{HC}^{13} \mathrm{~N}$, and $\mathrm{HCN}^{15} \cdot \Delta-\Delta$ transitions of both DCN and HCN in the $\nu_{3}$ fundamental regions have been analyzed. The splitting of the $\Delta$ levels was clearly observed for the first time and is in agreement with the $l$-type resonance calculations. The line structure of the $Q$ branches of both $\pi-\pi$ and $\Delta \nu \Delta$ "hot bands" has also been resolved. By means of the Ritz principle the levels $2 \nu_{2}^{0}, 2 \nu_{2}^{2}$, and $\nu_{2}^{1}$ of $\mathrm{DCN}$ and $2 \nu_{2}^{2}$ of $\mathrm{HCN}$ have been determined.

Ellipsometry in the measurement of surfaces and thin films, Symposium Proceedings Washington 1963, ed. E. Passaglia, R. R. Stromberg, and J. Kruger, NBS Misc. Publ. 256 (Sept. 15, 1964), \$2.25.

A symposium on the Ellipsometer ana its Use in the Measurement of Surfaces was held at the National Bureau of Standards in September 1963. This volume contains 19 of the papers included in the program, together with any discussions which followed oral presentation. Topics covered include bistorical review, theory, computational techniques, measurement techniques, and the use of ellipsometry in measuring metal surface oxide films and organic films.

Research on cyrstal growth and characterization at the National Bureau of Standards January to June 1964, ed. H. F. McMurdie, NBS Tech. Note 251 (Oct. 19, 1964), 45 cents.

The National Bureau of Standards with partial support from the Advanced Research Projects Agency of the Department of Defense is continuing a wide program of studies involving crystalline materials. These include investigation of methods and theory of growth, study of detection and effects of defects, determination of physical properties, refinement of chemical analysis, and determination of stability relations and atomic structure. The types of materials range from organic compounds, through metals, and inorganic salts to refractory oxides. This Technical Note, the fourth in the series, summarizes the progress of these various projects from January to June, 1964, and lists the related publications and participating scientists.

Procedures for precise determination of thermal radiation properties November 1962 to October 1963, J. C. Richmond, D. P. DeWitt, and W. D. Hayes, Jr., NBS Tech. Note 25\% (Nov. 20, 1964), 40 cents.

The preliminary design of an intergrating-sphere reflectometer, utilizing a helium-neon continuous-wave gas laser as the source, for measuring the reflectance of specimens at high temperature, was completed. Development work on an ellipsoidal mirror reflectometer for measuring spectral reflectance in the wavelength range of 2 to 15 microns of specimens at room temperature was continued. The study of equations relating spectral emissivity of metals to other properties was continued. Platinum- $13 \%$ rhodium and oxidized Inconel working standards of normal spectral emittance were calibrated over the wavelength range of 1 to 15 microns at temperatures of 800,1100 and $130{ }^{\circ} \mathrm{K}$. Several modifications of the normal spectral emittance equipment were made to permit operation in the $15-35$ micron range.

Franck-Condon factors for the ionization of $\mathrm{CO}, \mathrm{NO}$, and $\mathrm{O}_{2}$, M. E. Wacks, J. Chem Phys. 41, No. 4, 930-936 (Aug. 1964). Calculated values of the vibrational overlap integrals for the following transitions are given:

a) $\mathrm{CO}\left(\mathrm{X}^{1} \Sigma^{+}, \mathrm{v}^{\prime \prime}=0\right) \rightarrow \mathrm{CO}^{+}\left(\mathrm{X}^{2} \Sigma^{+}, \mathrm{v}^{\prime}=0 \rightarrow 10\right)$; $\left(\mathrm{A}^{2} \pi_{\mathrm{i}}, \mathrm{v}^{\prime}=0 \rightarrow 10\right) ;\left(\mathrm{B}^{2} \Sigma^{+}, \mathrm{v}^{\prime}=0 \rightarrow 10\right)$.

b) $\mathrm{NO}\left(\mathrm{X}^{2} \pi, \mathrm{v}^{\prime \prime}=0\right) \rightarrow \mathrm{NO}^{+}\left(\mathrm{X}^{1} \Sigma^{+}, \mathrm{v}^{\prime}=0 \rightarrow 10\right) ;\left(\mathrm{A}^{1} \pi, \mathrm{v}^{\prime}=0 \rightarrow\right.$ $10)$.

c) $\mathrm{O}^{2}\left(\mathrm{X}^{3} \Sigma^{-}{ }_{\mathrm{g}}, \mathrm{v}^{\prime \prime}=0,1\right) \rightarrow 0_{2}+\left(\mathrm{X}^{2} \pi_{\mathrm{g}}, \mathrm{v}^{\prime}=0 \rightarrow 10\right) ;\left(\mathrm{a}^{4} \pi_{\mathrm{u}}\right.$, $\left.\mathrm{v}^{\prime}=0 \rightarrow 15\right) ;\left(\mathrm{A}^{2} \pi_{\mathrm{u}}, \mathrm{v}^{\prime}=0 \rightarrow 20\right) ;\left(\mathrm{b}^{4} \Sigma^{-}{ }_{\mathrm{g}}, \mathrm{v}^{\prime}=0 \rightarrow 10\right)$

These probabilities are used to construct theoretical ionization efficiency curves assuming an energy dependence for the electronic transition integral for both photoionization and ionization by electron impact. Previously published experimental data are discussed in terms of the theoretically calculated Franck-Condon probabilities.

Versatile Stark waveguide for microwave spectroscopy, D. R. Lide, Jr., Rev. Sci. Instr. 35, No. 9, 12\%6 (Sept. 1964). A simple waveguide design for Stark-modulation microwave spectrometers is described. The guide provides a homogeneous Stark field which is perpendicular to the microwave 
electric field. The design has been used successfully in spectrometers operating at high frequencies, bigh temperatures, and for other special purposes.

Alkaline earth cation distributions in various borates, S. Block and G. J. Piermarini, Phys. and Chem. of Glasses 5, No. 5, 138-144 (Oct. 1964).

X-ray scattering curves of two different strontium borate glasses have been measured and from these curves, electron radial distribution functions have been calculated. The resulting distribution curves are compared with earlier work on barium borate, barium borosilicate, and cadmium borate. The curves are found to be all strikingly similar which indicates a corresponding similarity in the short-range order of the heavy cations for each glass. An analysis of the distribution curves based on the random packing of hard spheres suggests that the heavy cations occupy characteristic positions in the overall glass structure. These positions are governed to a certain extent by crystal chemical restraints as is demonstrated by a correlation between distribution curves obtained both from the glass and corresponding polycrystalline material.

Microwave spectroscopy, D. R. Lide, Jr., Ann. Rev. Phys. Chem. 15, 225-250 (1964).

This article is a critical review of research in microwave spectroscopy of gasses during the past several years. The major topics covered are accurate structure determinations, structures of new or unusual molecules, internal rotation, vibrational information, high temperature spectroseopy, and instrumentation. A bibliography of the most important publications is included.

Growth spirals on $\mathrm{NiBr}_{2}$ platelets, E. N. Sickafus and D. R. Winder, J. Appl. Phys. 35, No. 8, 2541-2545 (Aug. 1964). Platelets of $\mathrm{NiBr}_{2}$ have been grown by sublimation in an atmosphere of water vapor and helium. Several crystal habits were observed including dendrites, platelets, and whiskers. Platelets and dendrites grow in the region of substrate temperatures greater than $500{ }^{\circ} \mathrm{C}$ and although these crystals are anhydrous the presence of water vapor greatly enhances the growth rate. The crvstals are deliquescent. In the early stages of water adsorption the surface is not wetted but water droplets form at surface imperfections. The resultant decoration of surface morphology exhibits an anisotropy related to the surface structure. Photomicrographs are presented of concentric growth spirals occurring on the isolated faces of the same crystal platelet. These structures are demonstrated via direct observation of the opposite sides of the crystal, superposition of the opposite sides in thin regions of growth, and with the aid of Fizeau interferograms.

Infrared spectrum of $\mathbf{C F}_{2}$, D. E. Milligan, D. E. Mann, and M. E. Jacox, J. Chem. Phys. 41, No. 5, 1199-1203 (Sept. 1, 1964).

A low temperature infrared study of the photolysis products of $\mathrm{CF}_{2} \mathrm{~N}_{2}$ in inert matrices has yielded the three vibrational fundamentals of the species $\mathrm{CF}_{2}$, at 668, 1102, and 1222 $\mathrm{cm}^{-1}$. Supplementary ultraviolet observations have confirmed the presence of $\mathrm{CF}_{2}$ in high concentration. Detection of the stretching fundamentals of $\mathrm{C}^{13} \mathrm{~F}_{2}$, present in natural abundance, has permitted an estimate of the FCF angle. The thermodynamic properties of $\mathrm{CF}_{2}$ have also been estimated. $\mathrm{CF}_{2}$ appears not to react with $\mathrm{CO}$ or with $\mathrm{CO}_{2}$ in a matrix environment.

Change in the heat capacity of boron trioxide during the glass transformation, S. S. Chang and A. B. Bestul, J. Phys. Chem. 68, 3082 (1964).

A previous compilation showed that for $\mathrm{B}_{2} \mathrm{O}_{3}$ the change in heat capacity during the glass transformation, $\Delta c_{p}$, is only about one fifth of the "universal" value which applies to most glass-forming substances. A computational error has been found in the value for $\mathrm{B}_{2} \mathrm{O}_{3}$ in the above-mentioned compilation. The correct value of $\Delta c_{p}$ for $\mathrm{B}_{2} \mathrm{O}_{3}$ is 2.65 cal. deg. ${ }^{-1}$ per "bead", which agrees very well with Wunderlich's "universal" $\Delta c_{p}$ value of $2.7 \pm 0.5$ cal. deg. ${ }^{-1}$. Thus
$\mathrm{B}_{2} \mathrm{O}_{3}$ in fact behaves like other glass-forming substances in this respect, rather than being exceptional.

The structure of the triclinic form of sodium tetrametaphosphate tetrahydrate, H. M. Ondik, Acta Cryst. 1\%, No. 9, 1139-1145 (Sept. 1964)

The cell dimensions of triclinic sodium tetrametaphosphate tetrahydrate, $\mathrm{Na}_{4} \mathrm{P}_{4} \mathrm{O}_{12} \cdot 4 \mathrm{H}_{2} \mathrm{O}$, are $a=6.652 \pm 0.001, \quad b=$ $9.579 \pm 0.002, \mathrm{c}=6.320 \pm 0.001 \AA, \alpha=103^{\circ} 24^{\prime} \pm 1^{\prime}, \beta=106^{\circ} 59^{\prime}$ $\pm 1^{\prime}, \gamma=93^{\circ} 17 \pm 1^{\prime}$, space group $P \overline{1}, \mathbf{V}=1$.

The structure was determined from a three-dimensional Patterson function, and refined by complete matrix least squares. The $\mathrm{R}$ value for 1335 observed non-zero reflections is 0.086 .

The tetrametaphosphate ring is on the center of symmetry at the origin of the unit cell. One of the two crystallographically different $\mathrm{Na}$ ions is five-fold coordinated and the other is six-fold coordinated.

The five-fold coordinated $\mathrm{Na}$ ion links the rings along $c$, the six-fold coordinated ion links the rings along $a$. There are some similarities between the structures of the monoclinic and triclinic forms of this salt but the differences are too great to permit a transition between the two forms in the solid state. Such a transition, reported by other workers, has not been confirmed by this investigator.

Standards of electromotive force, W. J. Hamer, J. Wash. Acad. Sci. 54, 297-315 (1964).

The history of the unit of electromotive force (emf) as maintained in the United States is reviewed. The method by which the unit is maintained by standard cells is discussed. Characteristics of standard cells are briefly considered. Data on the units of emf, as maintained in various countries, are given for the international comparisons made since 1948 . Brief discussions of voltage ranges, ac voltages, and zener diodes are included as they relate to emf standards.

Experimental transition probabilities for six oxygen multiplets, J. E. Solarski and W. L. Wiese, Phys. Rev. 135, No. 5A, A1236-A1241 (Aug. 31, 1964).

The transition probabilities of six oxygen I multiplets in the visible and near infrared region have been measured in a wall stabilized high current are operating either in pure oxygen or in a nitrogen-oxygen mixture. Temperatures were obtained from intensity measurements of an oxygen or nitrogen line of known transition probability and by applying the arc equilibrium and conservation equations. Several independent runs were carried out. The results agree usually within $20 \%$ with those of other experimental and theoretical methods.

Electrochemical calorimetry III. Thermal effects of nickelcadmium batteries, W. H. Metzger, Jr., and J. M. Sherfey, Electrochem. Technology 2, No. 9-10, 285-289 (Sept.-Oct. 1964 ).

This paper reports some interesting sidelights obtained while making calorimetric measurements during various chargedischarge cycles of nickel-cadmium cells proposed as satellite power sources by the National Aeronautics and Space Administration (NASA). Typical curves of heat changes as a function of time during operation are shown and discussed. The heat of reaction of oxygen with the constituents of the cell was measured and close agreement was obtained with the literature value for the heat of formation of cadmium oxide.

Electrochemical calorimetry. IV. Measurement of the Peltier heat and voltage attending the passage of current across a liquid junction, A. Brenner and S. L. Gray, $J$. Electrochem. Soc. 111, No. 11, 1225-1230 (Nov. 1964).

The experiments involved the measurement of the temperature change resulting from the passage of current across the interface of two electrolytes. Measurements were made at various distances from the junction so as to yield a temperature profile of the effect. The total heat effect was calculated from the integrated area under the curve of temperature profile. The coefficient obtained by dividing the total heat effect in joules by the current in coulombs was reported as a voltage equivalent.

The following values were obtained for three systems of electrolytes and are probably correct to within about $7 \mathrm{mv}$ : 
$0.04 M$ hydrochloric acid and $0.4 M$ hydrochloric acid, $30 \mathrm{mv}$. $0.90 \mathrm{M}$ hydrochloric acid and $3.0 \mathrm{M}$ potassium chloride, $80 \mathrm{mv}$. $1.15 \mathrm{M}$ hydrochloric acid and $1.21 \mathrm{M}$ lithium chloride, $80 \mathrm{mv}$.

A hypothesis was advanced to relate the voltage equivalent of the heat effects to the diffusion liquid junction potentials and to the Peltier effect.

The National Bureau of Standards tritiated water standards, W. B. Mann, J. Appl. Rad. and Isotopes 15, 628, Pergamon Press Ltd. (1964).

This letter notes the very close agreement obtained by Euratom and the National Bureau of Standards in their calibration, by gas counting, of the National Bureau of Standards tritiated water standard.

Diffusion in a polymer with lamellar morphology, polyethylene, R. K. Eby, J. Appl. Phys. 35, No. 9, 2720-2724 (Sept. 1964).

Data for the diffusion of ethane in polyethylene are presented together with the results of optical microscopy and x-ray diffraction for the same samples. These data support the hypothesis that diffusion in polvmers with a lamellar morphology occurs in the lamellar boundaries and that, therefore, lamellar orientation is an important parameter. Lamellar thickness and perfection are also important.

Lasers for length measurement, A. G. McNish, Science 146, No. 3541, 177-182 (Oct. 1964).

CW lasers will find extensive use in length measurement because of the high coherence and intensity of the light they produce.

Infrared spectrum and structure of gaseous $\mathbf{A l}_{2} \mathrm{O}$, M. J. Linevsky, D. White, and D. E. Mann, J. Chem. Phys. 41, No. 2, 542-545 (July 15, 1964).

The infrared absorption spectrum of $\mathrm{Al}_{2} \mathrm{O}$ trapped in solid argon and krypton matrices has been observed. It is possible from the observed spectra and isotope shifts for $\mathrm{Al}_{2} \mathrm{O}^{16}$ and $\mathrm{Al}_{2} \mathrm{O}^{18}$ to conclude that the molecular symmetry of $\mathrm{Al}_{2} \mathrm{O}$ is $\mathrm{C}_{2} \nu$ and that the $\mathrm{Al}-\mathrm{O}-\mathrm{Al}$ angle is probably not far from $145^{\circ}$. Force constants and the bending frequency $\nu_{2}$ have been calculated. Reanalysis of the ultraviolet spectra of exploding aluminum wires provides additional support both for the bent structure and for the calculated value of $\nu_{2}$. The Al-O bond distance is estimated to be $1.66 \mathrm{~A}$. The 3 fundamental frequencies for $\mathrm{Al}_{2} \mathrm{O}^{16}$ in an argon matrix are (in $\mathrm{cm}^{-1}$ ) : $\nu_{1}=715, \nu_{2}=[238] ; \nu_{3}=994$.

High resolution infrared spectra of $\mathrm{C}_{2}{ }^{12} \mathrm{H}_{2}, \mathrm{C}^{12} \mathrm{C}^{13} \mathrm{H}_{2}$ and $\mathbf{C}_{2}^{13} \mathbf{H}_{2}$, W. J. Lafferty and R. J. Thibault, J. Mol. Spectry. 14, No. 1, 79-96 (Sept. 1964).

Two parallel bands in Fermi resonance, $\nu_{3}$ and $\nu_{2}+\nu_{4}{ }^{1}+\nu_{5}{ }^{1}$, have been studied in the $3200-3400 \mathrm{~cm}^{-1}$ region of $\mathrm{C}_{2}{ }^{12} \mathrm{H}_{2}$ The calculated unperturbed frequencies for $\nu_{3}$ and $\nu_{2}+\nu_{4}{ }^{1}+\nu_{5}{ }^{1}$ are $3288.66 \mathrm{~cm}^{-1}$ and $3288.11 \mathrm{~cm}^{-1}$, respectively. The assignment of $\nu_{3}$ to the higher frequency transition is confirmed by the study of this diad in $\mathrm{C}^{13} \mathrm{C}^{12} \mathrm{H}_{2}$ and $\mathrm{C}_{2}{ }^{13} \mathrm{H}_{2}$. In addition the absorption spectra of $\mathrm{C}^{13} \mathrm{C}^{12} \mathrm{R}_{2}$ and $\mathrm{C}_{2}{ }^{13} \mathrm{H}_{2}$ have been obtained in the $2600-2800 \mathrm{~cm}^{-1}, 3300-3400$, and $6400-6600 \mathrm{~cm}^{-1}$ spectral regions. The unperturbed vibrational frequencies have been obtained for $\mathrm{C}^{13} \mathrm{C}^{12} \mathrm{H}_{2}$, and a few of these frequencies have been calculated for $\mathrm{C}_{2}{ }^{13} \mathrm{H}_{2}$. Equilibrium B values have been obtained for the isotopic carbon molecules, and these values have been used to calculate accurate $r_{e}$ distances for acetylene. The "substitution structure" has also been calculated.

Rotation-vibration interaction and other corrections to the spin doublet separation in ${ }^{2} \Pi$ diatomic molecules, T. C. James, J. Chem. Phys. 41, No. 3, 631-641 (Aug. 1, 1964). The effect of rotation-vibration interaction on the spin doublet separation in ${ }^{2} \Pi$ states of diatomic molecules is treated. Almy and Horsfall's refinement of Hill and Van Vleck's treatment is questioned and an alternative approach making use of Dunham's treatment of ${ }^{1} \Sigma$ diatomic molecules is used. The $J$ dependence of the spin-orbit coupling con- stants is included in the discussion. Vibrational wave functions (including rotation-vibration interaction) and matrix elements are discussed. Additional corrections arising from the $\mathbf{I}$ doubling and from the spin-rotation interaction $\Gamma N \cdot S$ are briefly considered. A comparison of the results of this work with experimental spin doublet separation leads to better agreement between theory and experiment for the examples treated.

\section{Other NBS Publications}

J. Res. NBS 69B (Math. and Math. Phys.), No. 1 (Jan.Mar. 1965), 75 cents.

Lectures on matroids. W. T. Tutte.

Menger's theorem for matroids. W. T. Tutte.

Single-element extensions of matroids. H. H. Crapo.

Minimum partition of a matroid into independent subsets. J. Edmonds.

Lehman's switching game and a theorem of Tutte and Nash-Williams. J. Edmonds.

Character subgroups of $F$-groups. M. I. Knopp and M. Newman.

A note on multipliers of difference sets. R. A. Brualdi.

Modification of Edmonds' maximum matching algorithm. C. Witzgall and C. T. Zahn, Jr.

On measurable sets and functions. A. J. Goldman.

Characteristic formulation for nonperiodic solutions of Mathieu's equation. T. Tamir and H. C. Wang. On the surface duality of linear graphs. J. Edmonds.

Maximum matching and a polyhedron with 0,1-vertices. J. Edmonds.

J. Res. NBS 69C (Eng. and Instr.), No. 1 (Jan.-Mar. 1965), 75 cents.

Two picnometers of increased convenience and precision. A. Johnson.

Construction of a Michelson interferometer for Fourier spectroscopy. H. N. Rundle. (See above abstracts.)

The National Bureau of Standards gas thermometer II. Measurement of capacitance to a grounded surface with a transformer ratio-arm bridge. L. A. Guildner and R. E. Edsinger. (See above abstracts.)

An adiabatic calorimeter for the range, 10 to $360{ }^{\circ} \mathrm{K}$. K. F. Sterrett, D. H. Blackburn, A. B. Bestul, S. S. Chang, and J. Horman. (See above abstracts.)

A rugged null-type pressure transducer of high reproducibility for accurate gas phase PVT measurements. M. Waxman and W. T. Chen.

Detection and damping of thermal-acoustic oscillations in low-temperature measurements. D. A. Ditmars and G. T. Furukawa.

NBS free-air chamber for measurement of 10 to $60 \mathrm{kV}$ x rays. P. J. Lamperti and H. O. Wyckoff.

A compensated solenoid giving a uniform magnetic field over a large volume. C. Snow and R. L. Driscoll.

Evaluation of a microwave phase measurement system. D. A. Ellerbruch.

Polarographic analysis of titantium (IV)-EDTA complex: application to paint pigments. H. W. Berger and B. C. Cadoff.

Soil resistivity as related to underground corrosion and cathodic protection. W. J. Schwerdtfeger.

Radio Sci. J. Res. NBS/USNC-URSI, 69D, No. 2 (Feb. 1965). $\$ 1.00$.

Electromagnetic wave penetration of reentry plasma sheaths. M. P. Bachynski.

On the use of refractive index diagrams for source-excited anisotropic regions. L. B. Felsen.

Surface waves along a perfectly conducting plane covered with semi-infinite magneto-plasma. S. Adachi and $\mathrm{Y}$. Mushiake.

On the point of emergence of a microwave beam entering a linearly graded plasma. A. L. Cullen.

Self and mutual admittances of waveguides radiating into plasma layers. J. Galejs.

Effect of electron collisions on the formulas of magneto-ionic theory. K. G. Budden. (See above abstracts.) 
Momentum transfer collisions in oxygen for thermal electrons. M. H. Mentzoni.

Experimental studies of perturbations in ionospheric plasma. L. H. Heisler.

Electromagnetic scattering by gyrotropic cylinders with axial magnetic fields. W. C. Y. Lee, L. Peters, Jr., and C. H. Walter.

Radar cross sections of plasma bodies at the plasma frequency. L. Peters, Jr.

Electroacoustic waves excited by a space vehicle in ionized atmosphere and its effect on radar return. K.-M. Chen.

Discussion on basic equations with source terms in compressive plasmas. K.-M. Chen. (See above abstracts.)

Scattering of electromagnetic and electroacoustic waves by a cylindrical object in a compressible plasma. J. R. Wait.

Multiphase periodic very-low-frequency emissions. N. Brice.

Radio studies of the high-latitude ionosphere during the solar eclipse of 20 July $1963 . \quad$ R. D. Hunsucker.

VLF and LF fields propagating near and into a rough sea. R. M. Lerner and J. Max.

Insulated and loaded loop antenna immersed in a conducting medium. R. H. Williams.

Capacitance of biconical antennas in magneto-ionic media; elliptic cone capacitance. V. P. Pyati and H. Weil.

Calculations of the bistatic scattering cross section of a sphere with an impedance boundary condition. J. R. Wait and C. M. Jackson.

Concept of differential reflectivity as applied to the reflection of beam-limited radiation by a convex body. A. Erteza, J. A. Doran, and D. H. Lenhert.

Radio Sci. J. Res. NBS/USNC-URSI, 69D, No. 3 (Mar. 1965), $\$ 1.00$.

Propagation in nonuniform gyrotropic media. S. H. Gross and L. B. Felsen.

Geometrical optics for gyrotropic bodies. W. C. Y. Lee, L. Peters, Jr., and C. H. Walter.

Attenuation of hydromagnetic waves in the ionosphere. S.-I Akasofu.

Self distortion of radio signals in the $D$ region. L. R. Megill

Atmospheric gravity waves: a new toy for the wave theorist. C. O. Hines.

Electromagnetic fluctuations in an equilibrium plasma. R. E. Burgess.

A generalized hydromagnetic wave in an inhomogeneous, cylindrical plasma. C. K. MeLane and T. Tsukishima.

Angular dependence of the refractive index in the ionosphere. G. A. Deschamps.

Electrodynamies of moving anisotropic media: the first-order theory. C. T. Tai

Study of the phenomenon of whistler echoes. T. Laaspere, W. C. Johnson, and J. F. Walkup.

Multiple-frequency investigations of radio wave absorption during the dawn-breakup phase of auroras. R. Parthasarathy, and F. T. Berkey.

Sferic excitation of a two-layer conducting medium. Martin B. Kraichman.

Currents, charges, and near fields of cylindrical antennas. R. W. P. King and T. T. Wu.

A note on the radiation conductance of an axial slot on a cylinder. C. M. Knop and C. T. Swift.

Analytical formulas for radio paths in spherically stratified ionospheres. E. Woyk (Chvojkova).

A complete mode sum for LF, VLF, ELF terrestrial radio wave fields, J. R. Johler and L. A. Berry, NBS Mono. 78 (Oct. 1, 1964), 20 cents.

A study of lunar surface radio communication, L. E. Vogler, NBS Mono. 85 (Sept. 14, 1964), 70 cents.

Standard Reference Materials: Preparation of NBS copperbase spectrochemical standards, R. E. Michaelis, L. L. Wyman, and R. Flitsch, NBS Misc. Publ. 260-2 (Oct. 15, 1964), 35 cents.

Quarterly radio noise data June, July, August, 1963, W. Q. Crichlow, R. T. Disney, and M. A. Jenkins, NBS Tech. Note 18-19 (Aug. 21, 1964), 50 cents.

Some modifications in methods of calibration of universal ratio sets, D. Ramaley, NBS Tech. Note 220 (Aug. 30, 1964), 25 cents.
Concerning the theory of radiation from a slotted conducting plane in a plasma environment, J. R. Wait, NBS Tech. Note 223 (Sept 28, 1964), 20 cents.

Bibliography on the measurement of bulk resistivity of semiconductor materials for electron devices, J. C. French, NBS Tech. Note 232 (Oct. 21, 1964), 60 cents.

A nomogram for computing $\frac{a+j b}{c+j d}$ and a nomogram for computing $\left|\frac{a+j b}{c+j d}\right|$, H. S. Bowman, NBS Tech. Note 250 (Oct. 2, 1964), 15 cents.

Standards and measurement of attenuation, impedance and phase shift, R. W. Beatty, Proc. URSI XIVth General Assembly, Tokyo, Japan, Sept. 1963, XIII, 80-92 (1964). Vapour pressure, G. T. Armstrong, Encyclopaedic Dictionary of Physies $7,586-587$ (Pergamon Press Inc., New York, N.Y., 1962).

Analysis of a differential phase shifter, D. A. Ellerbruch, IEEE Trans. Microwave Theory Tech. MTT-12, No. 4 , 453-459 (July 1964).

Exterior corrosion of cast-iron pipe, M. Romanoff, J. Am. Water Works Assoc. 56, No. 9, 1129-1143 (Sept. 1964).

The energy loss of electrons in solids, A. M. Rauth and J. A. Simpson, Radiation Res. 22, No. 4, 643-661 (Aug. 1964). Coherence theories of tropospheric radio propagation, J. R Wait, IEEE Trans. Ant. Prop. AP-12, No. 5, 649-651 (Sept. 1964).

Solar activity, J. V. Lincoln, IQSY Instruction Manual. No. 2, Report of sudden ionospheric disturbances, Suppl., pp. 1-11 (IQSY Secretariat, London, England, 1963).

Comparison calibration of inductive voltage dividers, R. V. Lisle and T. L. Zapf, ISA Trans. 3, No. 3, 238-242 (July 1964).

IQSY Instruction Manual. No. 1. World days, A. H Shapley, 32 pages, IQSY Secretariat, London, England (July 1963).

Heater lead problem in calorimetrv, D. C. Ginnings and E. D. West, Rev. Sci. Instr. 35, No. 8, 965-967 (Aug. 1964).

Aspects of the National Bureau of Standards instrumentation literature reference file, J. F. Mayo-Wells (ISA 19th Annual Conf. and Exhibit, New York, N.Y., Oct. 12-15, 1964), ISA Preprint 20.6-5-64 (1964).

Influence of the refractive index profile in $\mathrm{VHF}$ reflection from a tropospheric layer, J. R. Wait and C. M. Jackson, IEEE Trans. Ant. Prop. AP-12, No. 4, 512-513 (July 1964).

Photometric observations of the airglow during the IQSY, F. E. Roach, IQSY Instruction Manual. No. 5: Airglow, Pt. I, 1-24 (IQSY Secretariat, London, England, 1963).

Correlation matrix for radio waves in the troposphere, E. C. Barrows (Proc. Symp. Electromagnetic Theory and Antennas, Copenhagen, 1962), Monograph, Electromagnetic Theory and Antennas 2, Sec. C, 743-746 (Macmillan and Co., Ltd., London, England, 1963).

Frequency and time broadcast services of the National Bureau of Standards, A. H. Morgan, Proc. ISA 17th Annual Automation Conf. and Exhibit, New York, N.Y., Oct. 15-18, 1962, Pt. I, Paper 1.1.62, 9 pages (1962).

Frostbite: signs, prevention, and treatment, J. R. MeyerArendt, Trail and Timberline, p. 3 (Jan. 1964).

Formation of an electron depleted region in the ionosphere by chemical releases, D. Golomb, N. W. Rosenberg, J. W. Wright, and R. A. Barnes (Proc. 4th Intern. Space Science Symp., Warsaw, June 4-10, 1963), Book, Space Research, ed. P. Muller, IV, 389-398 (North Holland Publ. Co., Amsterdam, The Netherlands, 1964).

The ionosphere, A. H. Shapley, U.S. Information Agency Voice of America Forum Series on Space Science, Lecture No. 7, 6 pages (1963).

Infrared spectroscopy of weak charge-transfer complexes, E. E. Ferguson, J. Chem. Phys. 61, 257-262 (1964).

Quarterly radio noise data September, October, November 1963, W. Q. Crichlow, R. T. Disney, and M. A. Jenkins, NBS Tech. Note 18-20 (Oct. 23, 1964), 50 cents.

Calculation of sunrise and sunset times at ionospheric heights along a great circle path, A. H. Brady and D. D. Crombie, NBS Tech. Note 209 (Nov. 8, 1964), 20 cents. 
A program for plotting circles of constant overpressure around targeted points, M. L. Joel and D. D. Lottridge, NBS Tech. Note 249 (Oct. 28, 1964), 40 cents.

Weights and measures - a challenge to industry, M. W. Jensen, Proc. 50th Mid-Year Meeting Chemical Specialties Manufacturers Assoc., Chicago, Ill., May 18-20, 1964, pp. 24-26 (Chem. Specialties Mfg. Assoc. Inc., New York, N.Y., 1964).

Interpretation of potentiometric titrations of weak acids in methanol-water solvents, K. C. Ong, R. A. Robinson, and R. G. Bates, Anal. Chem. 36, 1971-1972 (Sept. 1964).

Fluctuations in ionospheric absorption events at conjugate stations, J. K. Hargreaves and H. J. A. Chivers, Nature 203, No. 4948, 963-964 (Aug. 29, 1964).

RF and microwave power measurements, G. F. Engen, Proc. URSI XIVth General Assembly, Tokyo, Japan, Sept. 1963, XIII, 77-80 (1964)

LF-VLF frequency and time services of the National Bureau of Standards, D. H. Andrews (Proc. 19th Annual ISA Conf. and Exhibit, New York, N.Y., Oct. 12-15, 1964), ISA Preprint 21. 3-2-64 (1964).

Fresnel zone diffraction effects at $50 \mathrm{Gc} / \mathrm{sec}$. determined from measured aperture field data, R. C. Baird, Program and Digest 1963 IEEE Prof. Group Ant. and Prop. Symp. Space Telecommunications, July 9-11, 1963, Boulder, Colo., pp. 171-173 (1963).

Computer interpretation of English text and picture patterns, R. A. Kirsch, IEEE Trans. Electron. Computers EC-13, No. 4, 363-376 (Aug. 1964)

On the implication of diurnal, seasonal and geographical variations in composition of high atmosphere from $F$-region measurements, J. W. Wright (Proc. NATO Advanced Study Institute, Sheikampen, Norway, Apr. 17-26, 1963), Book, Electron Density Distribution in the Ionosphere and Exosphere, pp. 186-198 (North Holland Publ. Co., Amsterdam, The Netherlands, 1964).

Ionospheric winds: motions into night and sporadic $E$ correlations, N. W. Rosenberg, H. D. Edwards, and J. W. Wright (Proc. 4th Intern. Space Science Symp., Warsaw, June 4-10, 1963), Book, Space Research, ed. P. Muller, IV, 171-181 (North Holland Publ. Co., Amsterdam, The Netherlands, 1964).

Oblique incidence pulse measurement at $100 \mathrm{kc}, \mathrm{R}$. H. Dougherty, AGARDograph 74, Propagation of Radio Waves at Frequencies below 300 KC, pp. 133-148 (Pergamon Press Inc., New York, N.Y., 1963).

Nonresonant absorption and collision diameters in the foreigngas broadening of symmetric top molecules, G. Birnbaum and A. A. Maryott, J. Chem. Phys. 41, No. 1, 154-157 (July 1, 1964)

Methods of calibration of airglow photometers at Fritz Peak Observatory, L. L. Smith and R. B. Alexander, IQSY Instruction Manual No. 5: Airglow, Pt. II, pp. 25-36 (IQSY Secretariat, London, England, 1963).

Electromagnetic cross sections for electron and nuclear research, H. W. Koch, Nucl. Instr. Methods 28, 199-204 (1964).

Exclusion of parity unfavored transitions in forward scattering collisions, U. Fano, Phys. Rev. 135, No. 3B, B863-B864 (Aug. 10, 1964).

Microscopic-astrophysies and the development of astrophysical-laboratories, R. N. Thomas, Bull. Centre Intern. d'Ástrophysique de l'Observatoire de Nice, No. 1, pp. 7-16 (Nice, France, 1964).

Alouette ionic, magnetic-field and plasma studies, W. Calvert, H. Rishbeth, and T. E. VanZandt, IG Bull. No. 83, 16-19 (May 1964); Trans. AGU 45, No. 2, 398-401 (June 1964).

Ozonation of asphalt flux, P. G. Campbell and J. R. Wright, I. E. C. Prod. Res. and Devel. 3, 186-194 (Sept. 1964).

Propagation of radio waves past a coast line with a gradual change of surface impedance, J. R. Wait and K. P. Spies, IEEE Trans. Ant. Prop. AP-12, No. 5, 570-575 (Sept. 1964).

On the propagation of long wave-length terrestrial radio waves - two theoretical techniques, J. R. Johler and L. A. Berry, AGARDograph 74, Propagation of Radio Waves below $300 \mathrm{KC}$, pp. 101-129 (Pergamon Press Inc., New York, N.Y., 1963).

On the validity of the Einstein-Smoluchowski theory light scattering, R. Zwanzig, J. Am. Chem. Soc. 86, 3489-3493 (Mar. 30, 1964).

Microwave propagation in an overdense bounded magnetoplasma, B. Wieder, Phys. Fluids $\boldsymbol{\gamma}$, 964-972 (July 1964)

Pulsed and CW sinusoidal voltage and current measurements, M. C. Selby, Proc. URSI XIVth General Assembly, Tokyo, Japan, Sept. 1963, XIII, 92-100 (1964).

Thermodynamic data compilation and review at the National Bureau of Standards, G. T. Armstrong, Svensk Kem. Tidskr. 76, No. 8, 419-427 (1964).

Ionosonde studies of some chemical releases in the ionosphere, J. W. Wright (Proc. NATO Advanced Study Institute, Sheikampen, Norway, Apr. 17-26, 1963), Book, Electron Density Distribution in the Ionosphere and Exosphere, pp. 116-137 (North Holland Publ. Co., Amsterdam, The Netherlands, 1964).

Standard nuclear instrument modules, L. Costrell, U.S. Atomic Energy Commission TID Report 20893, 12 pages (July 1964).

Charge-transfer absorption spectra of $\mathrm{NO}$ in $\mathrm{Kr}$ and $\mathrm{CH}_{3} \mathrm{OH}$ solutions, E. E. Ferguson and H. P. Broida, J. Chem. Phys. 40, No. 12, 3715-3716 (June 15, 1964).

Ionospheric storms, S. Matsushita, Proc. URSI XIVth General Assembly, Tokyo, Japan, Sept. 1963, XIII, 169180 (1964).

Symplectic modulary groups, M. Newman and J. R. Smart, Acta Arithmetica IX, 83-89 (1964).

Propagation of a reflected shock wave in a transverse magnetic field, Y. Boublil and K. B. Earnshaw, Phys. Fluids 7 , No. 6, 876-881 (June 1964).

Behavior of coaxial cable connectors for pulses with nanosecond risetimes, R. B. Schwartz and A. C. B. Richardson, Nuclear Instruments and Methods 29, 83-88, NorthHolland Publ. Co. (1964).

Theory of boil-off calorimetry, R. B. Jacobs, Rev. Sci. Instr. 35, No. 7, 828-832 (July 1964).

"Thumbtack" accelerometer for the $1.5-150 \mathrm{kc}$ range, $\mathrm{P}$ Hertelendy and P. Reynard, Rev. Sci. Instr. 35, No. 10, 1305-1306 (Oct. 1964).

The application of Archimedes' principle to determination of the apparent volume of leather, J. R. Kanagy, J. Am. Leather Chem. Assoc. LIX, No. 11, 636-649 (Nov. 1964).

On the twilight sodium emission-1: Observations from a southern hemisphere station, M. Gadsden, Ann. Geophys. 20, No. 3, 261-272 (1964)

Spectra tristimulus values for the CIE $(u, v, w)$ uniform spacing system, I. Nimeroff, J. Opt. Soc. Am. (Letter to Editor) 54, No. 11, 1365-1367 (Nov. 1964).

Proton-transfer reactions between $\mathrm{H}_{3}{ }^{+}$and saturated hydrocarbons, P. Ausloos and S. G. Lias, J. Chem. Phys. 40, No. 12, 3599-3605 (June 15, 1964).

Resinography with $8-\AA$ X-ravs, S. B. Newman, Symp. on Resinographic Methods, ASTM Spec. Tech. Publ. No. 348, (1963).

Microwave spectrum of the SO radical, F. X. Powell and D. R. Lide, Jr., J. Chem. Phys. 41, No. 5, 1413-1419 (Sept. 1964).

Resonances of a spherical void in a compressible isotropic plasma, J. R. Wait, J. Geophys. Res. 69, No. 19, 4189-4191 (Oct. 1, 1964).

Evaluating computer systems analysis and design work in the Federal Government, W. H. Gammon, Book, Ideas for Management, Systems and Procedures 1964, 319-322 (1964)

Some remarks on microgrammars, W. C. Watt, Proc. Wash. Linguistics Club 2, No. 1, 13-17, (Oct. 26, 1964).

Microwave and high-frequency calibration services of the National Bureau of Standards, W. E. Larson, IEEE Trans. Microwave Theory Tech. M TT-12, No. 4, 480-482 (July 1964).

Determination of the propagation constants for ultrasonic waves in melting and molten polyethylene, R. K. Eby, J. Acoust. Soc. Am. 36, No. 8, 1485-1487 (Aug. 1964).

Radiation from sources immersed in compressible plasma media, J. R. Wait, Can. J. Phys. 42, 1760-1780 (Sept. 1964).

Conductance of copper $m$-benedisulfonate hexahydrate in $N$-methylpropionamide from 20 to $40^{\circ}$, T. B. Hoover, J. Phys. Chem. 68, No. 10, 3003-3008 (Oct. 1964). 
Polarized electrons and positrons by tagging technique, $\mathrm{H}$. Olsen and L. C. Maximon, Phys. Rev. Letters 13, No. 3, 112-114 (July 20, 1964).

Theory of whisker growth and evaporation, J. A. Simmons, R. L. Parker, and R. E. Howard, J. Appl. Phys. 35, No. 7, 2271-2272 (July 1964).

Mass spectrometric study of the production of methylamine from azomethane, M. E. Wacks, J. Phys. Chem. 68, No. 9, 2725-2726 (Sept. 1964).

Gaussian correlation functions: Two-electron systems, W. A. Lester, Jr., J. Chem. Phys. 41, No. 5, 1407-1413 (Sept. 1, 1964).

Nonspattering solder flux, W. R. Bjorklund, Rev. Sci. Instr. 35, No. 10, 1369-1370 (Oct. 1964).

Use of the index of refraction as a means for study of plasma configurations, E. G. Johnson, Phys. Fluids 7, 1551-1552 (Sept. 1964).

Application of the theory of absorbing Markov chains to the statistical thermodynamics of polymer chains in a lattice, J. Mazur, J. Chem. Phys. 41, No. 8, 2256-2266 (Oct. 15, 1964).
A combinatorial problem and a simple decoding method for cyclic codes, M. Nesenbergs, IEEE Trans. Inform. Theory IT-10, 250-252 (July 1964).

Observed line shapes of collective excitations in $\mathrm{Al}, \mathrm{Be}$, and Ge, N. Swanson, J. Opt. Soc. Am. 54, No. 9, 1130-1133 (Sept. 1964).

Flash-photolysis spectroscopy of matrices, L. J. Schoen and D. E. Mann, J. Chem. Phys. 41, No. 5, 1514 (Sept. 1964).

*Publications for which a price is indicated are available by purchase from the Superintendent of Documents, U.S. Government Printing Office, Washington, D.C. 20402 (foreign postage, one-fourth additional). Reprints from outside journals and the NBS Journal of Research may often be obtained directly from the authors. 